\title{
Emergent properties at oxide interfaces controlled by ferroelectric polarization
}

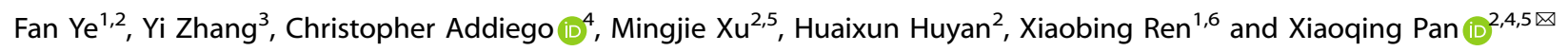

Ferroelectric materials are characterized by the spontaneous polarization switchable by the applied fields, which can act as a "gate" to control various properties of ferroelectric/insulator interfaces. Here we review the recent studies on the modulation of oxide hetero-/homo-interfaces by ferroelectric polarization. We discuss the potential applications of recently developed four-dimensional scanning transmission electron microscopy and how it can provide insights into the fundamental understanding of ferroelectric polarization-induced phenomena and stimulate future computational studies. Finally, we give the outlook for the potentials, the challenges, and the opportunities for the contribution of materials computation to future progress in the area.

npj Computational Materials (2021)7:130; https://doi.org/10.1038/s41524-021-00601-w

\section{INTRODUCTION}

Perovskite oxides have attracted extensive research interest due to their various adjustable order parameters and their mechanicalelectromagnetic coupling effects (Fig. 1) ${ }^{1-4}$. Among the perovskites, ferroelectric materials have spontaneous electric polarization that can be switched by external fields, which is fundamental to their numerous properties ${ }^{5-7}$. With the development of microelectronics technology, researchers are continually pursuing larger ferroelectric polarization and smaller form factors, leading to extensive research in epitaxial thin films ${ }^{8-11}$. In particular, heterointerfaces and domain walls (DWs) are becoming the emerging topological functional elements, and have attracted more and more attention.

A ferroelectric heterointerface is composed of two different materials, as shown in Fig. 1. These heterointerfaces sometimes exhibit intriguing physical properties, such as superconductivity $^{12,13}$, the tunneling electroresistance (TER) effect ${ }^{14-16}$, and highmobility charge-carrier gases ${ }^{17}$, which exceed the limitations of chemical contraindication in bulk materials ${ }^{18}$. In addition, polarization can be used as a "switch" to control the physical state (i.e., electric or magnetic properties) at the heterointerface; this is referred to as the "polar gating" effect ${ }^{19-24}$. For example, twodimensional electron gases (2DEG) and ferromagnetism may appear at the ferroelectric/insulator interface due to the discontinuous change in polarization and can also be modulated by changing the polarization state of the film ${ }^{12,13,17,25,26}$.

On the other hand, DWs in ferroelectrics can be recognized as a type of homogeneous interface (Fig. 1), which are twodimensional boundaries separating two regions (domains) with different polarization orientations, resulting in particular functional characteristics ${ }^{27}$. DWs have attracted increasing attention due to their functionalities distinct from the bulk, such as increased conductivity $^{28-31}$, magnetism ${ }^{32-34}$, and even superconductivity $^{35,36}$. Moreover, as ferroelectric DWs can be readily created, erased, and reconfigured within the same physical volume by external stimuli, they can be used as functional elements for the development of future nanoelectronic devices.
If we look closer into these different types of interfaces mentioned above, we can easily find that the highly localized properties are related to the polarization structure in ferroelectric materials. A polarization discontinuity exists both at the heterointerfaces and DWs, which induce electronic reconstruction at or near the interface and result in many properties ${ }^{26,37-46}$. The functional properties of ferroelectric materials are closely related to their microstructures and polarization configurations.

Transmission electron microscopy (TEM) allows us to investigate the functional properties of ferroelectrics from the perspective of the atomic structure, chemical composition, and simple bonding structure ${ }^{47-52}$. In broad terms, the imaging techniques applied to ferroelectrics can be divided into two categories: conventional diffraction-contrast imaging, including bright field/dark-field (BF/ DF) TEM, and aberration-corrected scanning transmission electron microscopy (AC-STEM).

Conventional TEM uses the parallel illumination condition where a broad parallel electron beam transmits through a thin specimen, forms a diffraction pattern in reciprocal space. Electrostatic lenses are then used to produce either the diffraction pattern or a reconstituted image of the sample in real space which can be recorded by a camera. Diffraction-contrast imaging in TEM is very sensitive to changes in the diffraction condition which can visually enhance the contrast of different ferroelectric domains at various scales. Since the entire field of view is continuously illuminated by the electron beam, this technique is ideal for in situ experiments that require high temporal resolution. If the instrument is equipped with in situ TEM specimen holders, this can enable the observation of dynamic domain structural change under external stimuli such as ferroelectric switching ${ }^{53-57}$.

On the other hand, the electron beam in STEM is converged on the sample to form a small probe and scan the area of interest to generate a pixelated map using the signal collected from each pixel position. In AC-STEM imaging, after the probe interacts with the sample and a convergent beam electron diffraction (CBED) pattern is projected onto annular detectors which generate different types of images depending on the scattering angle of the electrons. Several different types of secondary electrons, $X$ rays, plasmons, and other excitations can also be generated when

\footnotetext{
${ }^{1}$ Frontier Institute of Science and Technology, Xi'an Jiaotong University, Xi'an, Shaanxi, China. ${ }^{2}$ Materials Science and Engineering, University of California, Irvine, CA, USA. ${ }^{3}$ School of Physics, Sun Yat Sen University, Guangzhou, China. ${ }^{4}$ Department of Physics and Astronomy, University of California, Irvine, CA, USA. ${ }^{5}$ Irvine Materials Research Institute, University of California, Irvine, CA, USA. ${ }^{6}$ Center for Functional Materials, National Institute for Materials Science, Tsukuba, Japan. ${ }^{\circledR}$ email: xiaoqinp@uci.edu
} 


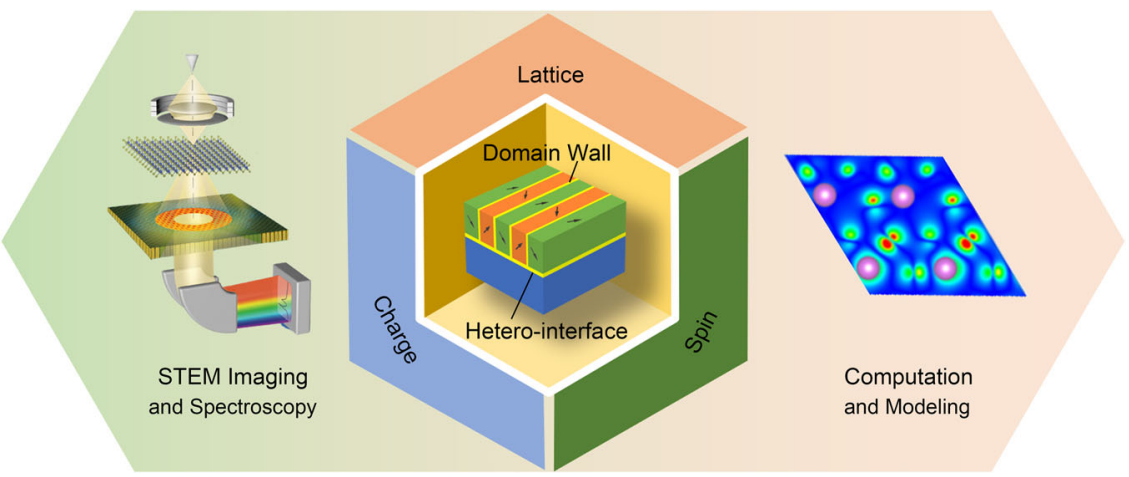

Fig. 1 The key properties and techniques of ferroelectric heterointerface and domain wall that were covered in this article. The heterointerface and DWs in ferroelectric and the electromagnetic properties coupled by the lattice distortion, spin polarization, and charge transfer. These characteristics are often studied by the combination of STEM imaging and spectroscopy, computational, and modeling techniques. Reproduced with permission from refs. ${ }^{200}$ and ${ }^{201}$. Copyright [2018] \{American Physical Society\} and [2018] \{American Association for the Advancement of Science\}.

the electron probe hits the sample; these can be analyzed either with dedicated detectors or examining the inelastic scattering of the electron beam. With the help of the aberration corrector, which reduces the spherical aberration introduced by the electromagnetic lenses, the size of the probe can reach the subAngstrom scale, which enables atomic-resolution imaging and mapping.

With such a high-resolution imaging method, we can quantitatively measure the atomic displacement and determine the polarization direction in ferroelectrics ${ }^{58-63}$. Through energydispersive spectroscopy (EDS) and electron energy-loss spectroscopy (EELS) technology, we can identify the type and concentration of elements and quantitatively analyze the defects. EELS can also tell us the change of valence state and charge transfer in the ferroelectric matrix or at interfaces ${ }^{49,64-66}$. If the readers are interested in this technique, we recommend ref. ${ }^{67}$, the detailed review article regarding the AC-STEM for atomic-resolution studies of functional oxides ${ }^{67}$.

Although these interesting interfacial phenomena can generally be visualized directly using various techniques nowadays, the fundamental principles are not so easily recognized. For example, when it comes to the detailed electronic band structure, properties such as the density of states at the sub-atomic level are difficult to study using conventional characterization techniques without the assistance of computational modeling. This is the reason why in the field of $2 \mathrm{D}$ topological structures, computational modeling always plays an essential role in terms of predicting emergent interfacial properties or build a bridge between the observation and the principle. In this article, we will not focus on the detailed explanation and introduction of various computational techniques, but rather demonstrate how these techniques, especially the first-principles calculation and phasefield simulation, are being applied in some of the most recent topics to complement experimental studies. For more information on the computational techniques, we encourage readers to check out these in-depth reviews and book chapters ${ }^{68-72}$.

Now with the development of a next-generation camera system, the electric field, and charge density at the subangstrom scale can be measured with four-dimensional scanning transmission electron microscopy (4D-STEM) which uses spatially resolved diffraction imaging data sets ${ }^{73-76}$. Combining in situ TEM and scanning probe microscopy (SPM), we can study the polarization dynamics, domain dynamics, and charge dynamics in ferroelectric materials at large spatial and temporal scales ${ }^{53,57,60}$. These emerging experimental techniques can provide more detailed input to the modeling as become great methods to validate the computational results, as shown in Fig. 1.
The review is structured as follows. In "Topological structure at ferroelectric heterointerfaces", we discuss the effects of polar gating on modulating the properties of ferroelectric heterointerfaces. We subsequently provide a critical review on the conductance of ferroelectric DWs in "Ferroelectric DWs". Finally, in "Discussion and perspective: 4D-STEM andbeyond", we review advanced techniques for investigating ferroelectric polarization and its functional properties at the atomic scale, e.g., 4D-STEM, which is followed by an outlook at the opportunities and challenges of ferroelectric from the perspective of characterization and modeling technologies.

\section{Topological structure at ferroelectric heterointerfaces}

Ferroelectric materials offer various physical properties in their heterostructures with metals, semiconductors, and insulators. More importantly, such interfacial properties can often be controlled by the polarization state of the ferroelectric film ${ }^{48,49,77}$. $\mathrm{Wu}$ et al. fabricated the first metal-ferroelectric-semiconductor transistor $^{78}$. This ferroelectric field-effect transistor (FeFET) uses the polarization in a ferroelectric gating layer to control a charge accumulation or depletion state even in the absence of an external field at the ferroelectric/Si interface, making it an ideal candidate for non-volatile memory devices ${ }^{24,78,79}$. In an analogous manner, the screening charge on the ferroelectric side of a polar/ non-polar interface can also be modulated ${ }^{80} .2 \mathrm{DEGs} s^{17,81-86}$ attract a lot of attention because of their unusually high electron mobility and other coupled phenomena such as ferromagnetism $25,26,42$, superconductivity $^{12,13}$, and quantum Hall effect ${ }^{87,88}$. The 2DEG at the $\mathrm{LaAlO}_{3} / \mathrm{SrTiO}_{3}$ (LAO/STO) interface is the prototypical example of which has been studied extensively since its discovery in $2010^{38,39,41,89}$. In ferroelectric-based oxide heterostructures, the polarization presents another avenue for controlling the properties of the 2DEG; complex and tunable two-dimensional electronic states can be achieved due to the switchable ferroelectric polarization $^{37,90,91}$.

Many theoretical works have been done trying to understand the origin of the 2DEG at oxide interfaces. Take LAO/STO as an example, one of the most intensively investigated mechanisms is called electronic reconstruction, which means that a charge of 0.5 electrons per unit-cell area $(\mathrm{e} / \mathrm{A})$ is formed at the interface to avoid the electrostatic potential divergence ${ }^{17}$. In this case, extrinsic doping such as oxygen vacancies becomes the perfect candidate because it can provide a wide range of carrier density depending on the configuration. Density-functional theory (DFT) calculations have shown that a low concentration of oxygen vacancies existed on the LAO surface can result in a 2DEG at the interface with a maximum density of 0.5 e/A (Fig. $2 a-c$ ), while as the density of 

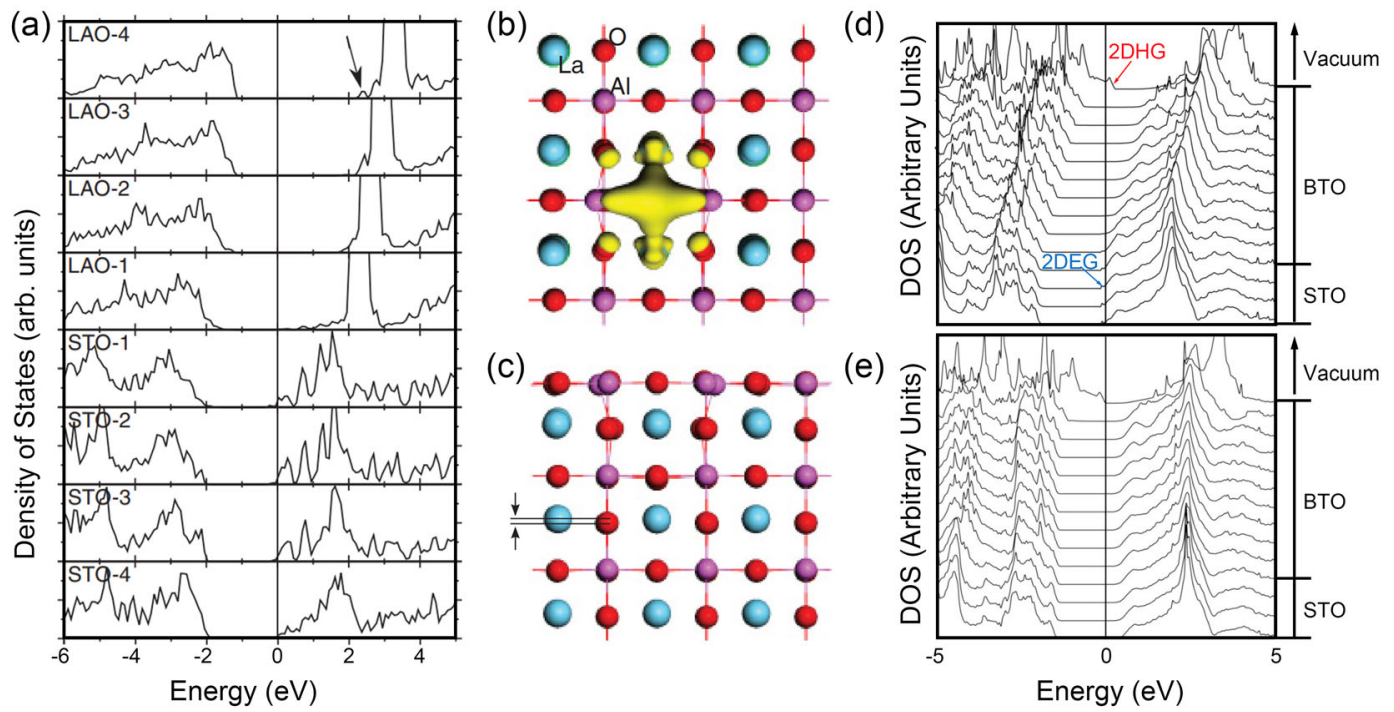

Fig. 2 Computational studies of the origin of the 2 DEG at oxide interfaces. a The DFT calculation of the layer-projected DOS of $(3 \times 3)$ supercell with one oxygen vacancy on the LAO surface. The arrow points to the oxygen-vacancy state. $\mathbf{b}$ Top view of the spatial distribution of the oxygen-vacancy state at the surface. c Side view of the top three LAO layers. The arrows and horizontal lines denote the ionic displacement in LAO. Reproduced with permission from ref. ${ }^{92}$. Copyright [2011] \{American Physical Society\}. d, e Layer-projected DOS for (d) $\mathrm{P}_{+}$and (e) $\mathrm{P}_{0}$ at a BTO/STO interface. Reproduced with permission from ref. ${ }^{84}$. Copyright [2015] \{American Physical Society\}.

oxygen vacancies increases, the thickness of the electron gas can extend into the STO substrate and become three dimensional ${ }^{92}$. However, this is not the only mechanism to explain the formation of 2DEG or two-dimensional hole gas (2DHG) at interfaces. Taking $\mathrm{BaTiO}_{3}$ (BTO)/STO as an example, DFT calculations found that thin $\mathrm{TiO}_{2}$-terminated $\mathrm{BTO}(<5 \mathrm{~nm})$ with $\mathrm{Ti}$ displaced toward the STO substrate $\left(P_{+}\right)$can form 2DEG at the interface and 2DHG at the surface (Fig. $2 \mathrm{~d}$ ) ${ }^{84}$. In this case, the electronic reconstruction forms two conducting layers on each side of the BTO film when electrons in the high-energy surface state migrate to the interface to avoid the polar catastrophe. Therefore, these conductive layers can easily diminish when the film thickness is high or the film becomes unpolarized $\left(P_{0}\right)$ (Fig. $\left.2 e\right)^{84}$. The latter mechanism enables the possibility of modulating the interface conductivity by changing the polarization state of the film.

\section{Polarization-induced interfacial 2DEG and the "polar-gating effect"}

When polarization points towards or away from the ferroelectric/ insulator interface, electrons or holes can accumulate at this interface. The accumulated charge may form 2DEG or 2DHG, depending on the types of the materials system as well as the polarization configuration $37,80,90,93,94$. In this case, the polarization acts as a "gate" to control the properties of ferroelectric heterojunction, which we call the "polar-gating effect". More interestingly, the 2DEG induced by ferroelectric polarization has also been shown to be ferromagnetic ${ }^{80}$, which means it is possible to control multiferroic properties by the polar-gating effect.

Based on first-principles band structure calculations using $\mathrm{KNbO} / \mathrm{ATiO}_{3}(\mathrm{~A}=\mathrm{Sr}, \mathrm{Ba}, \mathrm{Pb})$ model systems ${ }^{95}$, the 2DEG at the ferroelectric/oxide interfaces is switchable between two conduction states by the reversal of the ferroelectric polarization. The density of states (DOS) projected onto the Nb-4d states and Ti-3d states, which are located at different monolayers / away from the $\left(\mathrm{NbO}_{2}\right)^{+} /(\mathrm{SrO})^{0}$ interface is shown in Fig. 3a. The conduction band minimum is below the Fermi energy $\left(E_{\mathrm{F}}\right)$, and the electron density increases $/$ decreases. This indicates that a highly confined $n$-type conductive band, namely 2DEG is formed at the interface. Interestingly, the DOS and the number of occupied states are different at the two interfaces which have opposite polarization configurations (the left interface and right interface shown in Fig. $3 b)$. This predicts the possibility to create a $2 \mathrm{DEG}$ in $\mathrm{KNbO}_{3} / \mathrm{ATiO}_{3}$ $(\mathrm{A}=\mathrm{Sr}, \mathrm{Ba}, \mathrm{Pb})$, and the $2 \mathrm{DEG}$ is switchable between two conduction states as controlled by ferroelectric polarization orientation.

Experimentally, a similar switchable 2DEG has been observed in epitaxial $\mathrm{Pb}(\mathrm{Zr}, \mathrm{Ti}) \mathrm{O}_{3}(\mathrm{PZT}) / \mathrm{LAO} / \mathrm{STO}$ heterostructure ${ }^{37,94}$. Figure $3 \mathrm{C}$ shows the conductivity difference of the LAO/STO interface when the polarization of the PZT capping layer is changed. When the polarization direction of PZT is towards the LAO/STO interface (on state), the 2DEG is formed. While the polarization direction of the PZT is away from the LAO/STO interface (off state), the electron density is depleted and the conductivity decreases ${ }^{37,94}$. Wang et al., revealed the mechanism for this polarization-based modulation of the LAO/STO interface using EELS to examine the Ti fine structure across the interface, as shown in Fig. $3 \mathrm{~d}, \mathrm{e}^{94}$. Based on this analysis, the $\mathrm{Ti}^{3+}$ fraction at the interface of the bare LAO/STO and $\mathrm{PZT}_{\text {down }} / \mathrm{LAO} / \mathrm{STO}$ is approximately $37 \%$ and drops to zero after five unit cells. In contrast, no $\mathrm{Ti}^{3+}$ is detected in $\mathrm{PZT}_{\text {up }} / \mathrm{LAO/STO}$. The experimental results agree with the polar catastrophe model, that the $3 d$ band of titanium ion contains half an electron per Ti site, producing $\mathrm{Ti}^{3.5+}$ at the LAO/STO interface, and $\mathrm{Ti}^{4+}$ elsewhere ${ }^{96,97}$.

In another work, Zhang et al. found that the $\mathrm{BiFeO}_{3}(\mathrm{BFO}) /$ $\mathrm{TbSCO}_{3}$ (TSO) (001) interface is conducive along with the $109^{\circ}$

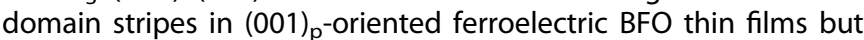
insulating in the direction perpendicular to this domain stripes ${ }^{48}$. Figure $4 a$ shows the PFM image of the cross-section BFO specimen in $(1 \overline{1} 0)_{\text {o }}$ plane, where the $109^{\circ}$ domain stripes structure can be observed. The corresponding conductive atomic force microscopy (CAFM) image is shown in Fig. 4b, which indicates the interface of BFO/TSO is conductive along the domain strips. Interestingly, the distribution of current is not homogeneous (Fig. 4c). The conductivity is higher when the polarization points towards the interface and lower when the polarization points away from the interface. However, according to Fig. $4 d$, e, the interface is non-conductive across the $109^{\circ}$ domain stripes.

To understand the origin of anisotropic conductivity at the interface, first-principles calculations were performed ${ }^{48}$. Figure $4 \mathrm{f}$ shows the atomic structure and corresponding layer-by-layer density of state (DOS) for the BFO/TSO (001) interface. 
(a)

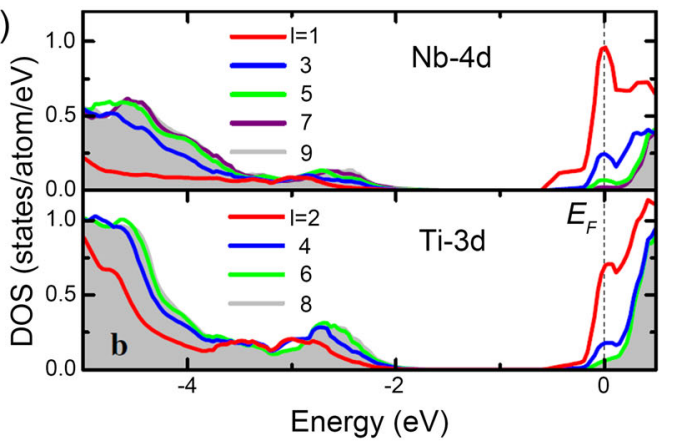

(c)

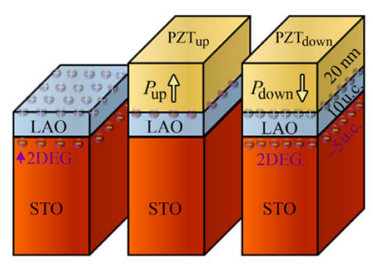

(d)

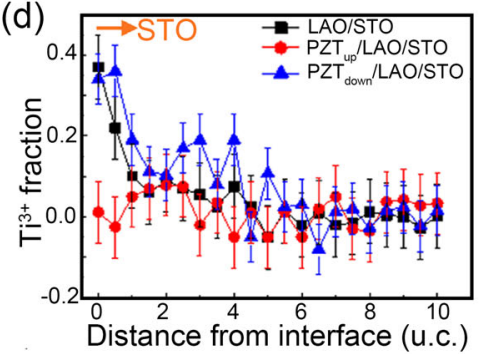

(b)
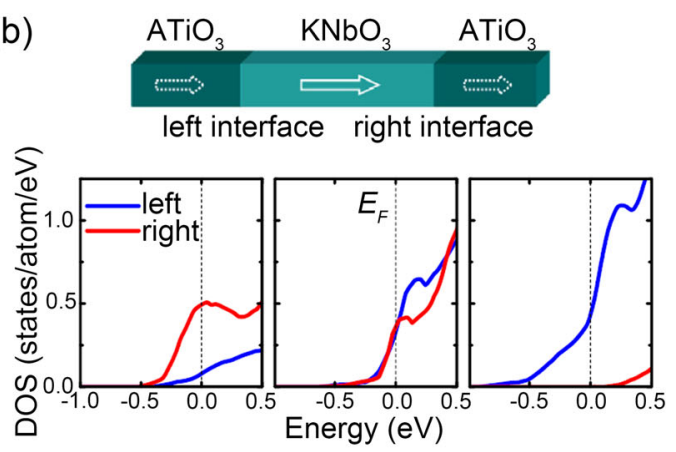

(e)

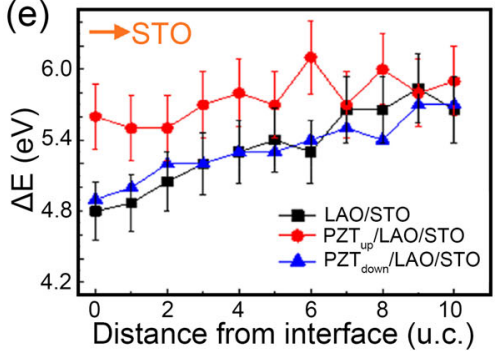

Fig. 3 Ferroelectric polarization modulated the conductivity at the heterointerface. $\mathbf{a}, \mathbf{b} \mathrm{KNbO}_{3} / \mathrm{ATiO} \mathrm{O}_{3}(\mathrm{~A}=\mathrm{Sr}, \mathrm{Ba}, \mathrm{Pb})$ heterointerface. The density of state (DOS) projected onto (a) Nb-4d states and (b) Ti-3d states, which are located at different monolayers $/$ away from the ( $\left.\mathrm{NbO}_{2}\right)^{+} /$ $(\mathrm{SrO})^{0}$ interface for a paraelectric state of $\left(\mathrm{KNbO}_{3}\right)_{8.5} /\left(\mathrm{SrTiO}_{3}\right)_{7.5}$ superlattice. Reproduced with permission from ref. ${ }^{95}$. Copyright [2009] \{American Physical Society\}. c-e $\mathrm{LaAlO}_{3} / \mathrm{SrTiO}_{3}$ heterointerface. c The schematic of bare LAO/STO, PZT interface. d, e Evolution of $\mathrm{Ti}^{3+}$ fraction and $\Delta E\left(\mathrm{O} \mathrm{K}\right.$-edge) with distance from the interface. Reproduced with permission from ref. ${ }^{94}$. Copyright [2018] \{American Chemical Society\}.

The ferroelectric polarization produces a rigid shift of the DOS of BFO, leading to the accumulation of electrons at interface 1 (polarization pointing towards the interface) and holes at interface 2 (polarization pointing away from the interface). These accumulated free carriers create either an n-type or p-type conductive interface, depending on the orientation of ferroelectric polarization. The polarization-controlled interface charge distribution is further clarified by phase-field simulation. The three-dimensional schematic model of the BFO domain structure with $109^{\circ} \mathrm{DWs}$ is shown in Fig. 4g. As shown in the bound charge distribution and electric potential profile shown in Fig. $4 \mathrm{~h}$, i, respectively, the alternating positive/negative charge build-up also caused the formation of $p-n$ junctions at the DWs, which blocks the conducting path perpendicular to the domain stripes, thus resulting in anisotropic conductivity.

\section{Polarization-induced interfacial ferromagnetism}

Multiferroics can realize the coexistence and mutual coupling of electricity and magnetism. In such systems, there is usually an interaction between the charges, spins, orbits, and crystal lattices. Therefore, various quantum phenomena can often be derived in magnetoelectric multiferroics or their composite structures ${ }^{87,88,98}$. This provides opportunities for the development and design of quantum computing devices. By using the electric field to control the magnetism in the future high-density information storage technology, the Joule thermal energy consumption caused by the writing process can be greatly reduced ${ }^{81,99-102}$.

Scientists have demonstrated local, high, and non-volatile carrier spin polarization in ferroelectric tunnel junction with ferromagnetic electrodes ${ }^{103}$. The reversible electrical switching of spin polarization can be controlled by the ferroelectric polarization of the barrier because of the coupling between ferroelectric polarization and magnetization $20,80,104$. Here, we focus on the polarization-controlled ferromagnetism at a heterointerface.

In 2018, Zhang et al., found that a spin-polarized (SP)-2DEG is formed at the non-magnetic insulating $\mathrm{SrTiO}_{3} / \mathrm{PbZr}_{0.2} \mathrm{Ti}_{0.8} \mathrm{O}_{3}$ (STO/
PZT) heterostructure, which was grown on the (110) $\mathrm{DyScO}_{3}$ (DSO) substrate, forming an STO/PZT/DSO structure ${ }^{80}$. According to the EELS spectra of STO near the STO/PZT(/DSO) interface, as shown in Fig. $5 a, b$, the emergence of a 2DEG originates from the $\mathrm{Ti}^{3+}$ at the interface. By analyzing the spectra differential conductance $(G(V))$ measured by Andreev reflection spectroscopy (ARS) collected from the interface, the interfacial metallic behavior can be confirmed with a spin polarization of $61 \%$, indicating that the interface is a spin-polarized SP-2DEG, as shown in Fig. 5c. Kerr effect analysis on the samples with various thicknesses of STO further confirmed that the ferromagnetism occurs only at the STO/ PZT interface (Fig. 5d).

To understand the origin of the SP-2DEG in the STO/PZT interface, first-principles calculations were performed. The calculations indicate that the magnetized interface states come from the interplay between band bending and local Coulomb interaction at the interfacial Ti sites. Theoretical calculations also revealed the possibility of switching the magnetic states via electric bias. The coexistence of high conductivity and a switchable magnetic state at the interface demonstrates a promising direction to design heterogeneous multiferroic materials and devices.

However, the ferromagnetic state in the STO/PZT heterointerface mentioned above can only be observed at very low temperatures, and cannot be switched into a different magnetic state. Recently, a ferromagnetic state was found at the $\mathrm{SrTiO}_{3} /$ $\mathrm{PbTiO}_{3}$ (STO/PTO) interface at room temperature which can be reversibly switched to antiferromagnetic by applying a magnetic field ${ }^{104}$. The high-angle annular dark-field scanning transmission electron microscopy (HAADF-STEM) image shows a sharp interface at the atomic level without obvious elemental interdiffusion (Fig. $5 e)$. Electron energy-loss spectra (EELS) for Ti were acquired across the STO/PTO interface, which indicates that the ratio of $\mathrm{Ti}^{3+}$ to $\mathrm{Ti}^{4+}$ at the interface is around $53.6 \%$. This result, combined with the lack of significant oxygen vacancy presence at the interface, confirms that electrons mainly accumulated at the interface. Scanning superconducting quantum interference device (SQUID) magnetometry $(50 \mathrm{kOe})$ shows that the STO/PTO heterostructures 
(a)

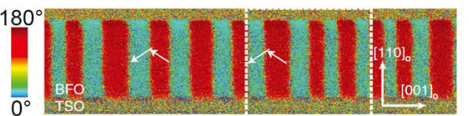

(b)

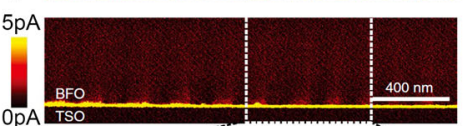

(c)

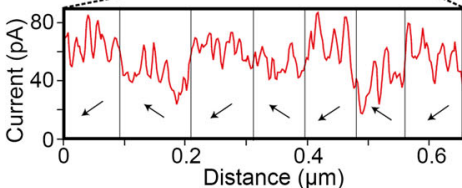

(d)

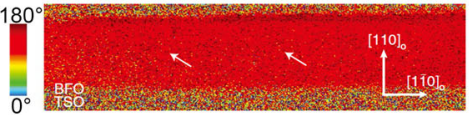

(e)

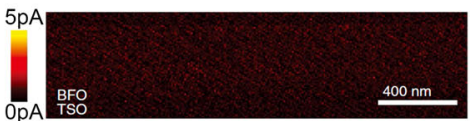

(f)
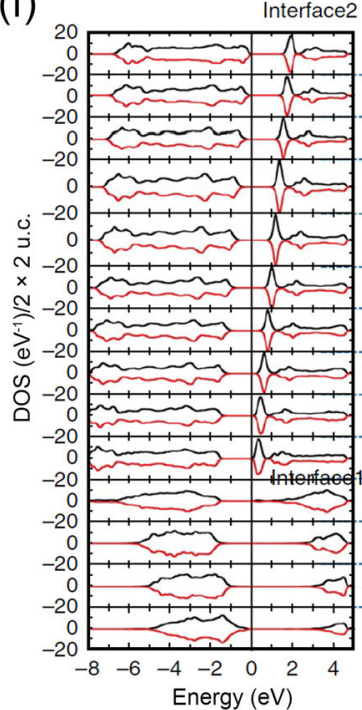

(g)

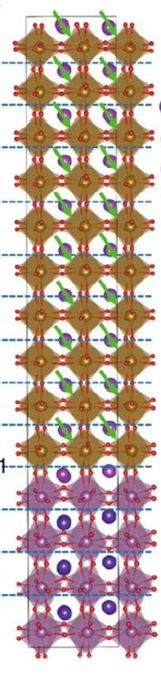

(h)

(i)
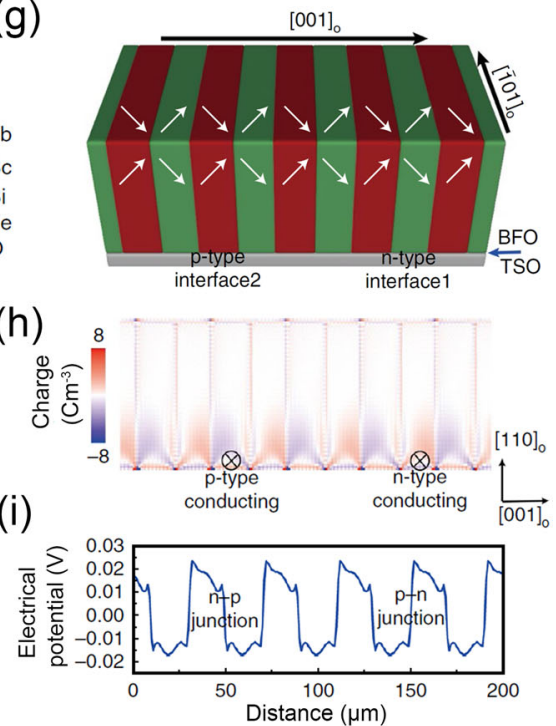

Fig. 4 Anisotropic conductance of 2DEG at the interface of BFO/TSO with $109^{\circ}$ domain arrays. a PFM image of BFO in a cross-section specimen perpendicular to the stripe domain. The directions of polarization are indicated by the white arrows. $\mathbf{b}$ CAFM image of the same region in (a). c Averaged current signal profile along the interface region marked by the white dashed line of (a). d PFM image of BFO in a cross-section sample parallel to the stripe domain. e CAFM image of the same region in (d), the interface is an insulator in this direction. $\mathbf{f}$ Layer-resolved density of state (DOS) and corresponding atomic structure for BFO/TSO (001) interface by first-principles calculations. $\mathbf{g}$ Threedimensional schematic model of BFO domain structure with $109^{\circ} \mathrm{DWs}$. $\mathbf{h}$ The distribution of bound charge at the BFO/TSO interface along with the domain stripes. The conduction of p-type and $n$-type are corresponding to interface 2 and interface 1 , respectively. $\mathbf{i}$ The distribution of electrical potential at the BFO/TSO interface perpendicular to the domain stripes. Reproduced with permission from ref. ${ }^{48}$. Copyright [2018] \{Springer Nature\}.

display an obvious ferromagnetic hysteresis below a critical magnetic field $\left(H_{\mathrm{T}} \sim 5 \mathrm{kOe}\right)$ at $300 \mathrm{~K}$ and can reversibly transition into diamagnetic behavior above $H_{\mathrm{T}}$. This suggests that the magnetic field-induced transition from ferromagnetic to diamagnetic is found at the interface (Fig. 5g). This interfacial ferromagnetism was further supported by high-resolutionelectron magnetic-chiral-dichroism (EMCD) measurements. As shown in Fig. 5f, obvious magnetic signals only occurred at the interface.

According to the experimental results, both itinerant electrons and local moments exist at the interface, and the itinerant electrons can be localized by applying the external field. Combined with the results of first-principles calculations, a mechanism was proposed where the interface-induced ferromagnetism and its reversible transition at the interface originate from the coupling between local moments $\left(\mathrm{Ti}^{3+}\right)$ and the itinerant electrons.

Many factors can affect the local behavior at heterointerfaces, which often makes finding the dominant underlying mechanism can be challenging for experimental or theoretical studies in isolation. The 2DEG that appears at the LAO/STO interface is one classic example where there still is no consensus on whether the primary cause is the polar catastrophe model, interfacial diffusion, or oxygen vacancies. But there are many other examples as well, such as the decreasing $\mathrm{Mn}$ valence at the $\mathrm{La}_{0.7} \mathrm{Sr}_{0.3} \mathrm{MnO}_{3}$ (LSMO) interfaces $^{105}$ and the degradation of the magnetic and transport properties in LSMO/STO multilayers ${ }^{106}$. The origins of these phenomena may be the imperfections such as intermixing at interfaces and variations in the A-site cation ratio, as well as oxygen vacancies ${ }^{105-108}$. The detection limits of TEM imaging and spectroscopy techniques often make it difficult to construct conclusive explanations for these phenomena based solely on experimental data. In these cases, integrated experimental and theoretical studies, where the issue is examined from multiple perspectives, can yield the greatest advances in the understanding of interfacial phenomena.

\section{Ferroelectric DWs}

Compared to the heterointerfaces, DWs are another functional element in ferroelectrics that have numerous applications in ferroelectric materials. Since ferroelectric DWs can be readily created, erased, and reconfigured by an external electric field, they can be used for non-volatile memory applications ${ }^{109-114}$. A fundamental understanding of the microscopic mechanisms behind these properties is critical for the design of practical devices. Extrinsically, point defects accumulated at DWs can create the interband states that lead to DW conductivity $28,29,115,116$. Also, the accumulated bound charge at "head-to-head $(\mathrm{H}-\mathrm{H})$ " or "tailto-tail $(T-T)$ " charged DWs (CDWs) gather compensating free charges, creating two-dimensional conducting channels, as shown in Fig. $6 a^{31,117-122}$. Intrinsically, the bandgap lowering at noncharged DWs, where no bound charge accumulated, is another source of the conductance, as shown in Fig. $6 f^{123-126}$.

Here, we focus on the conductivity of charged and non-charged DWs in ferroelectric thin films, which can be controlled by polarization.

\section{Polarization-controlled conductance of CDWs}

CDWs exhibit specific polarization discontinuities, such as $\mathrm{H}-\mathrm{H}$ and T-T configurations, and carry the net bound charge. They are electrically active and have a much higher conductivity than the charge-neutral DWs ${ }^{31,117-122}$. The polarization discontinuity at CDWs generates a local electric field which causes the absolute energies of the local electronic band structure to shift uniformly, as shown in Fig. $6 a^{116,127,128}$. The conduction bands dip below the Fermi Energy $\left(E_{\mathrm{F}}\right)$ at $\mathrm{H}-\mathrm{H}$ CDWs with n-type carriers, while the valence bands peak above $E_{\mathrm{F}}$ at T-T CDWs with p-type carriers.

The ferroelectric polarization can both induce conductance and also act as a gating mechanism to control the conductivity. Ma et al. controlled the conductance of square BFO nanoislands by switching the direction of polarization ${ }^{111}$. The structure of these BFO nanoislands is shown in Fig. $6 \mathrm{~b}$. This stable self-assembled 

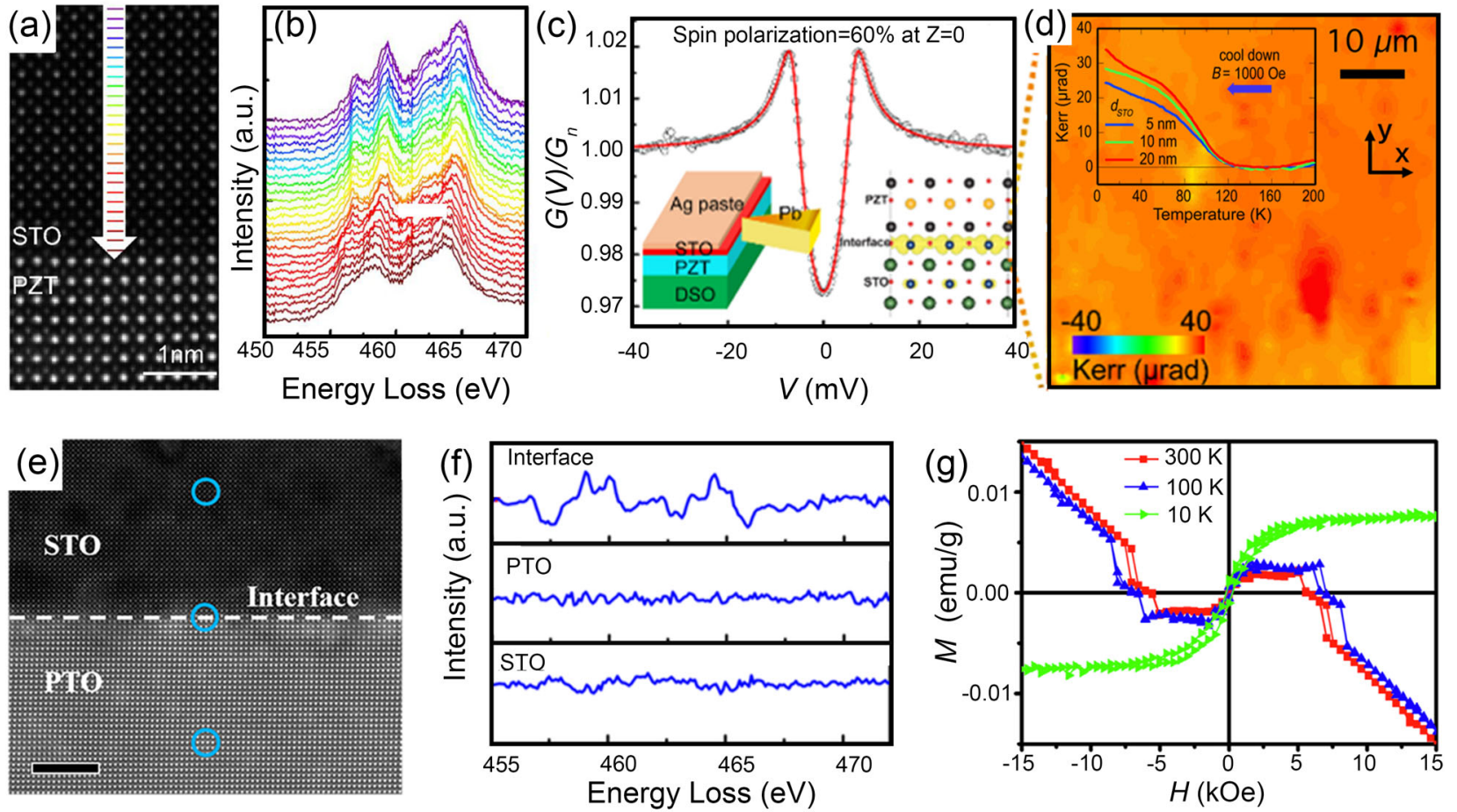

Fig. 5 Polarization-induced ferromagnetism at the ferroelectric/insulator interface. a-d The conductance of SP-2DEG at STO/PZT interface. a HAADF-STEM image and (b) corresponding EELS spectra in STO/PZT(/DSO) heterostructure. The broadened peaks in STO near the interface are attributed to the presence of $\mathrm{Ti}^{3+}$ near the STO/PZT interface. c Representative Andreev spectra (open circles) of point contact between Pb superconductor and STO/PZT interface with the best fits to the modified Blonder-Tinkham-Klapwijk (BTK) model (solid curves), where $T=$ $1.51 \mathrm{~K}, P=0.50, \Delta=1.31 \mathrm{meV}, r_{\mathrm{E}}=3.76, Z=0.21$, and $R=899 \Omega$. The inset is the schematic of point contact established at the STO/PZT interface. d 1000 Oe cool-down for three $(5,10,20 \mathrm{~nm}) \mathrm{STO} / 50 \mathrm{~nm}$ PZT/DSO sample and its Kerr signal image mapping at $4 \mathrm{~K}$ under 1000 Oe field, showing uniform ferromagnetism signal distribution. Scale bar, $15 \mu \mathrm{m}$. Reproduced with permission from ref. ${ }^{80}$. Copyright [2018] \{Springer Nature\}. e-g Ferromagnetism of STO/PTO heterointerface. e HAADF-STEM image of STO/PTO interface. The EMCD was taken in the marked areas (f). The EMCD patterns of STO/PTO heterointerface. $\mathbf{g} \mathrm{M}-\mathrm{H}$ curves of STO/PTO heterostructures at different temperatures. Reproduced with permission from ref. ${ }^{104}$. Copyright [2020] \{Elsevier\}.

center-convergent quad-domain system contains four $\mathrm{H}-\mathrm{H}$ CDWs. The domain evolution from a random initial state to this topologically stable state, as well as the switching process, was modeled by the phase-field simulation. Since the charge carriers in the LSMO bottom electrode are p-type, few free electron carriers can accumulate at the positively charged $\mathrm{H}-\mathrm{H}$ CDWs. As a consequence, these $\mathrm{H}-\mathrm{H}$ CDWs exhibit poor electrical conductivity according to the current map under a bias of $1.5 \mathrm{~V}$ in this initial state, (Fig. 6d). Once the CDWs transform to the negatively charged T-T DWs after polarization switching (Fig. 6c shows the center-divergent quad-domain configuration), the density of free hole carriers at the CDWs provided by the LSMO electrode is dramatically higher. Consequently, the current is also increased by approximately three orders of magnitude compared to the initial state when applying a $1.5 \mathrm{~V}$ bias, as shown in Fig. 6e. The ferroelectric polarization controls the conductivity of the square $\mathrm{BiFeO}_{3}$ nanoislands in a self-assembled array, which may have applications in the non-volatile memory industry with the nondestructive operation and ultralow energy consumption.

\section{The polarization-induced anisotropic conductance of non- charged DWs}

Besides the charged DWs, the non-charged DWs can also be conductive but the mechanism may be different. Seidel et al. first directly observe the conductivity at DWs in BFO; they found that $180^{\circ}$ and $109^{\circ} \mathrm{DWs}$ in multiferroic $\mathrm{BiFeO}_{3}$ thin films are conductive at room temperature while the $71^{\circ}$ DWs are non-conducting ${ }^{123}$. Numerous studies on DW conductivity have been carried out since then ${ }^{129,130}$. One mechanism of non-charged DW conductivity is from the inhomogeneous elastic strains. This strain gradient that exists near the DW regions decreases the local bandgap resulting in an increase in thermally excited conduction electrons, as shown in Fig. $6 f^{116,129,131}$. In addition, the polarization, created by the spontaneous strain gradient at the DW through the flexoelectric effect also introduces more free carriers ${ }^{116,131}$. Moreover, defects prefer to accumulate at DW regions, which will also increase the conductivity of DWs ${ }^{29}$. Tadej Rojac et al. directly confirmed the DW conduction in BFO originates from the accumulation of charged defects by atomic-scale chemical and structural analyses ${ }^{29}$. Large vertical lattice distortions across the DW come from a Bi sublattice shift in the direction parallel to the DW plane, resulting in vacancies at the DW. According to the energy onset difference $(\Delta E)$ between $\mathrm{O}-\mathrm{K}$ and $\mathrm{Fe}-\mathrm{L}_{3}$ edges, the oxidation of Fe on the DW region is +4 instead of +3 off the DW. The $\mathrm{Fe}^{4+}$ cations and bismuth vacancies act as charged defects screening polarization bound charges, revealing $p$-type hopping conduction at the DW. Thus, the DW conduction can be controlled by charged defects.

Interestingly, the polarization can induce the anisotropic conductance of non-charged DWs. The $71^{\circ} \mathrm{DWs}$ in the $\mathrm{BiFO}_{3} /$ $\mathrm{TbSCO}_{3}$ (BFO/TSO) thin-film are insulating in the direction perpendicular to the surface, which is consistent with other research results, as shown in Fig. $6 \mathrm{~g}, \mathrm{~h}^{123,129,132-134}$. However, these $71^{\circ}$ DWs are found to be conductive in $[010]_{p}$ direction, which is parallel to the thin-film surface, as shown in Fig. $6 i, j^{134}$. The conductivity of $71^{\circ} \mathrm{DWs}$ in $[010]_{p}$ stems from the polarizationinduced charge accumulation on the DWs. In the experiment, it was also observed that adjacent $71^{\circ}$ DWs exhibited alternating high and low conductivity. Through phase-field simulation, we can conclude that the conductivity difference of adjacent $71^{\circ} \mathrm{DWs}$ 
(a)

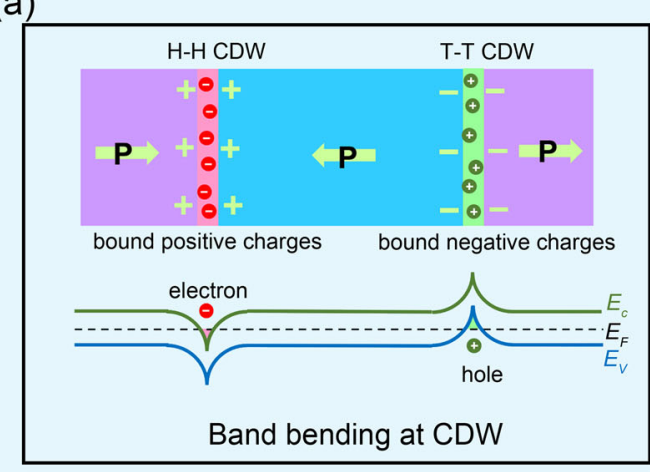

(f)

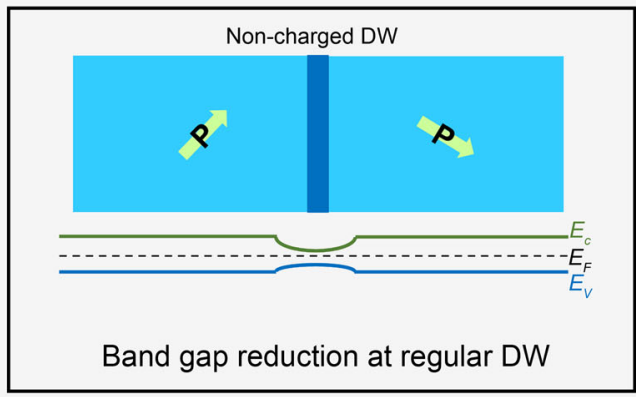

(b)

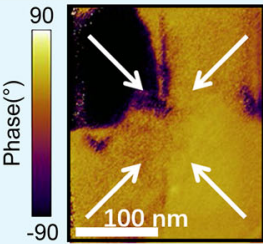

(d)

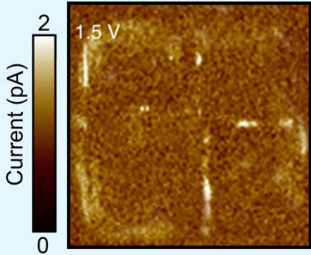

(g)

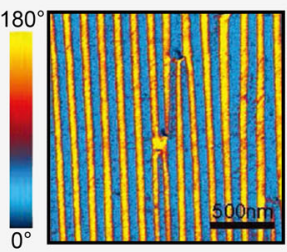

(c)

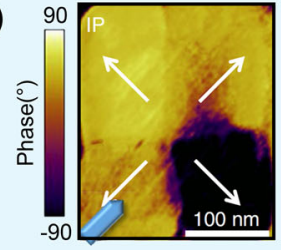

(e)

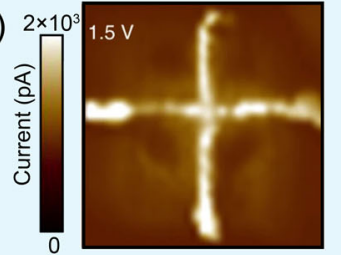

(h)

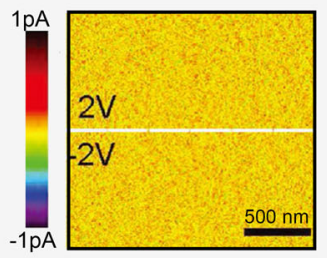

(i)

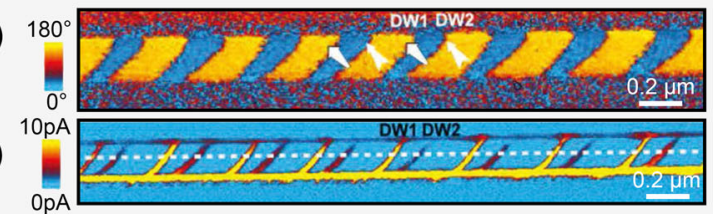

Fig. 6 Conductance of DWs. a-e The conductivity of charged DWs. a Mechanism of domain-wall conduction at charged DW originated by band bending. $\mathbf{b}$ In-plane (IP) PFM image of the BFO island at the initial state. The white arrows indicating the polarization directions in the center-convergent quad-domains. c The current map is under $1.5 \mathrm{~V}$ bias at the initial state. $\mathbf{d}$ In-plane (IP) PFM image of the BFO island after switching, which shows center-divergent quad-domain structur. e CAFM at the charged DWs under $1.5 \mathrm{~V}$ bias after polarization switching of the nanoislands. The switched DWs show large conductivity. b-d are reproduced with permission from ref. [111]. Copyright [2018] \{Springer Nature\}. $\mathbf{f}$-j Conductance at non-charged DWs. $\mathbf{f}$ Mechanism of conductance at non-charged DW originates from bandgap reduction. $\mathbf{g}$, $\mathbf{h}$ The $71^{\circ} \mathrm{DWs}$ of BFO are insulated in the directions normal to the surface. $\mathbf{g}$ Lateral PFM phase image showing $71^{\circ} \mathrm{DWs}$. $\mathbf{h}$ CAFM image of the same region in ( $\mathbf{g}) . \mathbf{i}, \mathbf{j}$ The $71^{\circ}$ DWs of BFO are conductive in the direction parallel to the surface. $\mathbf{i}$ PFM image of BFO in a cross-section specimen with $71^{\circ}$ DWs. The white arrows indicate the directions of polarization. $\mathbf{j}$ CAFM image of the same region in (i). $\mathbf{g}-\mathbf{j}$ are reproduced with permission from ref. ${ }^{134}$. Copyright [2019] \{John Wiley and Sons\}.

comes from the uneven width of the adjacent domains, as well as the electrostatic interaction between the walls across the narrow domains. The discontinuous potential distribution along the film thickness direction may induce a potential barrier along the wall and form a $\mathrm{p}-\mathrm{n}$ junction, which suppresses the conductivity across the film. The DW curvature can also enhance the potential discontinuity in the $71^{\circ} \mathrm{DW}$.

\section{Theoretical works on DW conductivity}

Although both charged and non-charged DWs have exhibited electrical conductivity, their conduction mechanisms are different $^{135}$. In order to clarify the mechanism of DW conductivity, many theoretical works have been performed ${ }^{115,133,136}$. For the conductivity of charged DWs, the recent theoretical work has shown that the driving force for the accumulation charge is due to the mismatch of the formal polarization between the constituent materials $^{136}$. These charges can induce $2 \mathrm{DEG}$ or $2 \mathrm{DHG}$ at the charged DWs, even when no oxygen vacancies, defects, or dopant atoms are introduced in their simulations (Fig. 7a, b). In addition, oxygen vacancies are considered to be an important factor affecting the conductivity of charged $\mathrm{DWs}^{137}$. First-principles calculations reveal that the interaction between charged DW and oxygen vacancies can enhance the conductivity of $\mathrm{H}-\mathrm{H}$ walls and reduces the conductivity of $\mathrm{T}-\mathrm{T}$ walls. This has been confirmed by experimental results showing that the conductivity of the $\mathrm{H}-\mathrm{H} \mathrm{DW}$ is higher than that of $\mathrm{T}-\mathrm{T}^{31}$. The main reason is that the screening charge at the $\mathrm{H}-\mathrm{H}$ DW is free electrons, while the screening charge at the T-T DW is an immovable oxygen vacancy. Therefore, the interaction between oxygen vacancies and charged DWs can modulate the conductivity of CDWs (Fig. 7c).

As more and more experimental results show that non-charged DWs are conductive ${ }^{28,134}$, additional theoretical studies have also emerged to explain why conventional DWs are conductive ${ }^{115,138}$. However, the mechanism of the non-charged DWs conductivity has seen considerable debate and remains a challenging problem due to the multitude of phenomena that may contribute, such as flexoelectric effect, octahedral rotation, oxygen-vacancy migration, chemical doping, DW geometry, and charged defects $28,29,124,139,140$. Some of the previous theoretical works using DFT for BFO DWs suggested that the DW conductivity in $\mathrm{BFO}$ originated from the lowering of the bandgap at the walls ${ }^{133}$, which is supported by the results from Seidel et al. ${ }^{28}$. However, discrepancies still exist between the theoretical results from different groups, for example, regarding the energy of different types of DWs ${ }^{133,141,142}$. A recent DFT work pointed out that the method used to study the electronic potential in the previous calculations was indirect and did not explicitly consider the excess charge carriers at DWs ${ }^{115}$. This work reveals that excess electrons are selectively trapped at pristine neutral DWs in BFO, while holes are only weakly attracted. Such trapped excess electrons may be responsible for the thermally activated electrical conductivity at DWs observed in experiments (Fig. 7e-g). 

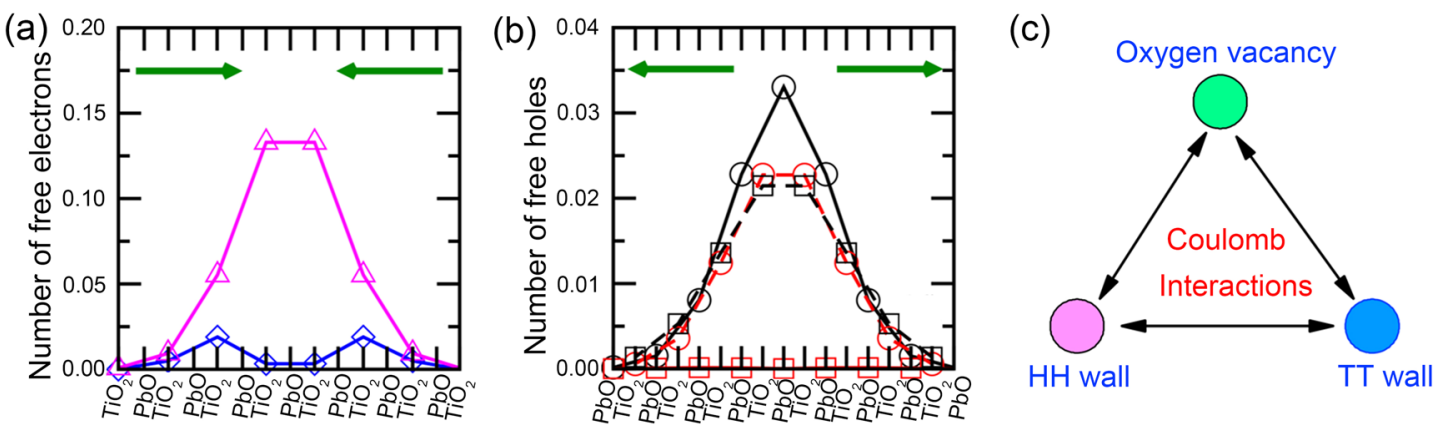

(d)
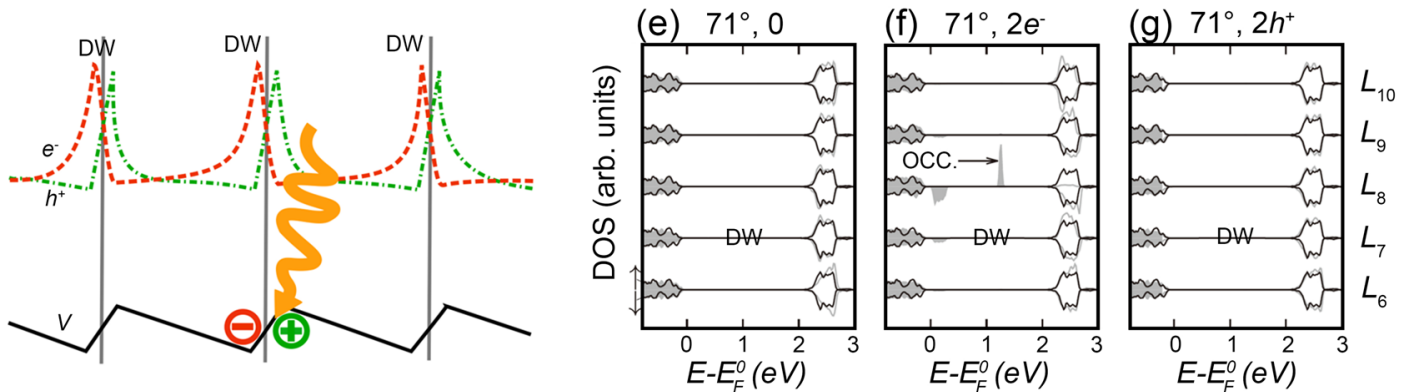

Fig. 7 Conductance of DWs. a, b Free-carrier densities in $\mathrm{PbTiO}_{3}$ for the domain wall in the (a) $\mathrm{H}-\mathrm{H}$ structure and (b) $\mathrm{T}-\mathrm{T}$ structure. Reproduced with permission from ref. ${ }^{136}$. Copyright [2020] \{American Physical Society\}. c A schematic representation of the interaction triangle between oxygen vacancy, $\mathrm{HH}$ (head-to-head) wall, TT (tail-to-tail) wall. Reproduced with permission from ref. ${ }^{137}$. Copyright [2018] \{Elsevier\}. d Model for the charge-carrier distribution of excess electrons $\left(\mathrm{e}^{-}\right)$, holes $\left(\mathrm{h}^{+}\right)$, and the electronic potential (V), at DWs. e- $\mathbf{g}$ The density of states (DOS) of the $71^{\circ} \mathrm{DW}$ system (gray) and the charge-neutral monodomain system (black) projected on the atomic layers. e Charge-neutral DW. $\mathbf{f}$ DW with electrons. $\mathbf{g}$ DW with holes. $\mathbf{d}-\mathbf{g}$ From Sabine Körbel et al. Panels $\mathbf{d}, \mathbf{g}$ are reproduced with permission from ref. ${ }^{115}$. Copyright [2018] \{American Physical Society\}.

Although the conductivity mechanism for non-charged DWs remains controversial, we believe that the factors influencing the conductivity can be divided into two categories: intrinsic and extrinsic, which can each play a leading role under certain conditions. Intrinsic factors include the potential step at the DW and the accompanying reduction in DW bandgap (Fig. 7d), which result in conductivity regardless of the exact atomic structure or chemical composition of the DW ${ }^{115,133}$. Extrinsic factors include oxygen vacancies, structural defects, and DW geometry and are most important for modulating the intrinsic conductivity of DWs and may be used to explore next-generation devices ${ }^{143}$. Therefore, only by making full use of factors that contribute to the mechanism for conductivity can the potential of DW functionalities be further released.

\section{Discussion and perspective: 4D-STEM and beyond}

The polarization is a fundamental order parameter of ferroelectrics, which can control the local charge or spin ordering across heterointerfaces. However, the charge-density distribution in the ferroelectric matrix or their interfaces is tough to obtain in the sub-angstrom scale with typical measurement methods. Recently, 4D-STEM/DPC-STEM (differential phase-contrast scanning transmission electron microscopy) was shown to be capable of measuring the real-space charge density with sub-angstrom resolution ${ }^{73}$. This technique can be used to elucidate the charge distribution across the DWs and ferroelectric/oxide interface. We present a brief introduction in this chapter.

Due to its versatility and fairly high spatial resolution (generally 5-10 nm), SPM has become the mainstream technology for researching the polar-gating effect ${ }^{18,144,145}$. S/TEM imaging can provide better spatial and temporal resolution to study atomicscale structures and intermediate stages of polarization switching $^{53,146}$, but conventional imaging methods still cannot be used to study some ferroelectric phenomena, such as charge screening. In order to study the polarization structure, a method sensitive to light elements and other electronic properties must be found. Atomic-resolution differential phase-contrast scanning transmission electron microscopy (DPC-STEM) ${ }^{74}$ is one such method that is sensitive to low- $Z$ species ${ }^{147}$ such as $\mathrm{O}^{148}, \mathrm{~N}^{149}, \mathrm{Li}^{150}$, and even $\mathrm{H}^{151}$, and is also sensitive to the local electronic properties of the material. The basic configuration of DPC is shown in Fig. 8a. As the electron beam is passing through the sample, it interacts with the local electric field via the Lorentz force. This causes a shift in the probe's momentum which is reflected in a shift in the intensity distribution of the CBED pattern. The CBED pattern is projected onto a set of segmented annular detectors and the shift in the CBED intensity is estimated based on the difference in the intensity detected by opposing segments of the detector. Therefore, it is possible to estimate the local electric field $(\mathbf{E})$ in the sample based on the DPC signal. The local charge density $(\rho)$ and electrostatic potential $(\mathbf{V})$ are related to the electric field via the following expressions from basic electrostatics:

$$
\frac{\rho}{\varepsilon_{\mathrm{o}}}=\boldsymbol{\nabla} \cdot \mathbf{E}
$$

$-\nabla \mathrm{V}=\mathbf{E}$

where $\varepsilon_{O}$ is the vacuum permittivity. Therefore, DPC data can be differentiated (dDPC) or integrated (iDPC) to generate images representing the charge density or potential, both of which can be used to identify the positions of light nuclei and the local electronic properties.

Campanini et al. mapped the polarization quantitatively at a strongly charged T-T DW of BFO by $\mathrm{APDC}^{152}$. The increased sensitivity for low-Z elements made it possible to visualize the oxygen vacancies, and the oxygen vacancies accumulate at the T-T CDW as the leading charge screening process, which can explain the enhancement conductance at the DW region.

4D-STEM is similar to the DPC technique mentioned above ${ }^{74,153-155}$, except that a fast camera is used to capture a complete $2 \mathrm{D}$ image of the CBED pattern for each position in the 
(a)

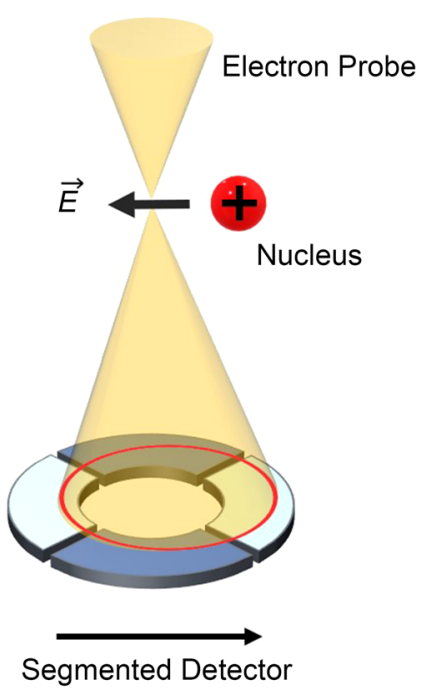

(b)

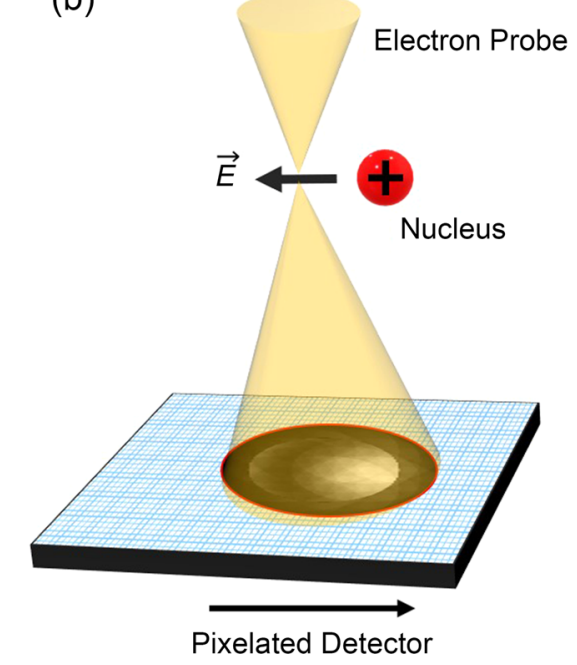

Fig. 8 Schematics depicting two different types of the setup for DPC and 4D-STEM. a DPC with a segmented detector and b 4D-STEM with a pixelated detector. When the electron probe passes through the electric field pointing left, the intensity in the diffraction pattern shifts right.
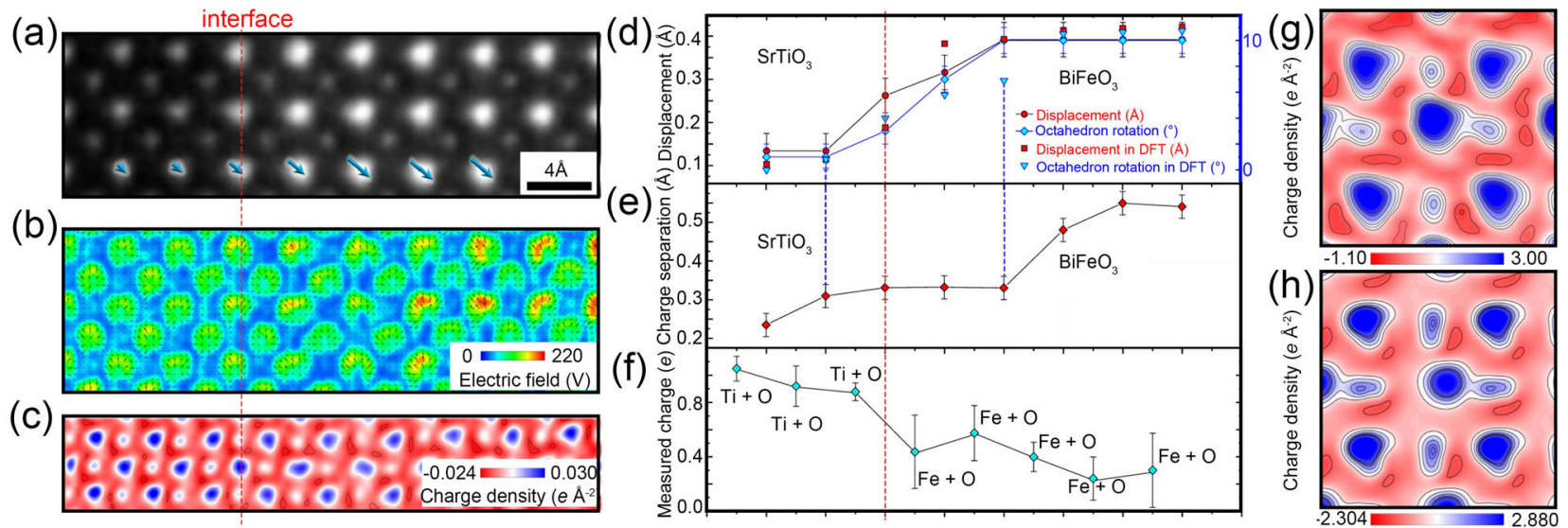

Fig. 9 4D-STEM applications to ferroelectricity. a A HAADF image from an STO-BFO interface; arrows indicate Bi displacement direction and magnitude. $\mathbf{b}, \mathbf{c}$ The corresponding E-field and charge-density maps were calculated from 4D-STEM data. $\mathbf{d}$-f Analysis of the atomic structure (d), charge separation (e), and integrated B-site charge (f) determined from the HAADF image and charge-density map. $\mathbf{g}$ Charge-density image of bulk BFO from scanning diffraction experiments (h). Charge-density image obtained from DFT calculations. Reproduced with permission from ref. ${ }^{73}$. Copyright [2019] \{Springer Nature\}.

raster scan instead of using annular detectors, resulting in a $4 \mathrm{D}$ dataset ${ }^{156}$. Figure $8(\mathrm{~b})$ shows the basic configurations of 4D-STEM. With this set-up, the change in the intensity distribution of the CBED pattern can be quantified by calculating the change in center of mass $(\boldsymbol{\Delta C O M})$. In very thin samples, with some approximations, the electric field can be calculated directly from $\Delta$ COM by the following expression:

$$
\mathbf{E}_{x y}=-\frac{\Delta \operatorname{COM} p_{z} v_{z}}{e \Delta z}
$$

where $p_{z}$ is the electron momentum along the beam direction, $v_{z}$ is the speed of the electron along the beam direction, $e$ is the charge of an electron, and $z$ is the sample thickness ${ }^{73,157}$. Though 4D-STEM cannot image in real-time like DPC, 4D-STEM has many applications outside electric field imaging. While the collection angles of conventional STEM images are limited by the detectors installed in the microscope, 4D-STEM data can be used to reconstruct images with arbitrary collection angles using "virtual" detectors that can be changed with software ${ }^{158-160}$. The orientation map can also be constructed from 4D-STEM data ${ }^{161,162}$.
Strain measurements based on CBED higher-order Laue zone images (HOLZ) ${ }^{163,164}$, and nanobeam electron diffraction are also variations of $4 \mathrm{D}$-STEM that are widely used. For a complete introduction to 4D-STEM, we refer the reader to ref. ${ }^{156}$.

When measuring the electronic properties of materials at interfaces or boundaries where physics emerges, such as the polar-gating effect in ferroelectric materials, the traditional techniques still cannot achieve sub-angstrom. Recently, Gao et al. imaged local electric fields and charge density in STO/BFO heterojunctions in real space with a high spatial resolution by state-of-art AC-STEM and 4D-STEM ${ }^{73}$. Figure 9a shows a HAADF image of the interface with the polarization gradually decreasing across the interface. Using a $4 \mathrm{D}$ dataset, the electric field is calculated at each scanning position based on the change in the probe's momentum (Fig. 9b); the charge density is then calculated based on the electric field (Fig. 9c). Based on the charge density, the separation of the positive and negative charge within the BFO unit cell can be calculated, revealing the electric dipole based on the full charge distribution. The charge state of each atomic column was also determined by integrating the charge within a 
region defined by Bader charge analysis. By tracking changes in the cation displacement, oxygen octahedral tilt, charge distribution, and charge state of the B-site column across the BFO/STO interface, as shown in Fig. 9d-f, Gao et al. were able to show how an asynchronous change in the atomic structure and the electron distribution led to a build-up of charge at the interface ${ }^{73}$. Although many other imaging techniques have shown that ferroelectric polarization can control charge build-up at ferroelectric interfaces, a mechanistic explanation of the polar-gating effect requires the sub-angstrom resolution provided by 4D-STEM. By combining high-resolution images of the full atomic structure (cations and anions) with images of the charge distribution, 4DSTEM can reveal the interfacial charge distribution in ferroelectrics with great detail. In addition, a high-resolution 4D-STEM provides an efficient method for measuring the detailed charge distribution within individual unit cells with sufficient detail that can be compared with DFT calculations. Whereas previous methods involving X-ray diffraction or quantitative convergent beam electron diffraction relied on computationally intensive structure-factor fitting to determine the charge distribution within a unit cell ${ }^{165-167}$, the charge density in 4D-STEM is calculated directly from each CBED pattern with a relatively simple analysis $^{157,168}$. As shown in Fig. $9 \mathrm{~g}$, $\mathrm{h}$, the charge density within a BFO unit cell measured by Gao et al. with 4D-STEM bears a close resemblance to the charge density calculated from $\mathrm{DFT}^{73}$. With the help of emerging 4D-STEM methods, we can now directly observe the detailed charge distribution at interfaces and boundaries. In addition, it is challenging to calculate the potential, electric field, and charge density from first principles for irregular structures such as defects and interfaces in the materials at an atomic scale because of the complexity of the structures and the increase of simulation time. The DPC and 4D-STEM experimental results provide direct measurements of these variables on the irregular structures, giving a better understanding of the structures' physics and thus can be used as guidance for the relevant simulations. By combining 4D-STEM with simulation, firstprinciple calculation methods, and advanced scanning probe methods, one can better understand the charge density and interface electronic/magnetic states at a more fundamental level. Furthermore, if 4D-STEM can be utilized during in situ experiments under different external fields, such as a magnetic field or electric field, it could be a powerful tool to solve many mysteries in the field of ferroelectrics.

DPC-STEM/4D-STEM does have some limitations. To quantitatively determine the electric field and charge density, the sample thickness must be less than about a few nanometers, much thinner than general STEM specimens $s^{51,73,169}$. Thicker specimens may cause channeling or beam broadening that will reduce the quantitative accuracy of the electrostatic properties determined with this method. In some circumstances, thicker samples can be used, but these generally require additional support from image simulations $s^{75,169}$. Generally, qualitative characteristics, such as the atomic positions and relative intensities, can be maintained in thicker samples with proper probe defocus ${ }^{51}$. Also, since the smallest achievable electron probe sizes are generally large compared to the length scale over which the electrostatic potential changes, the electric field measured in 4D-STEM/DPCSTEM is the electric field of the sample convolved with the shape of the electron probe. Aberration correction can ensure that the probe shape is rotationally symmetric to minimize any shape changes in the measured electric field caused by convolution with the probe. In addition, in regions with weaker electric fields, slightly displaced from the nuclear cores or around lighter elements, the electric field does not change over such small length scales and the probe convolution will have a smaller impact.

A combined multi-modal approach to studying ferroelectric interfaces that fully utilize SPM, S/TEM imaging, spectroscopy, and
4D-STEM can simultaneously reveal functional properties and the underlying physical mechanisms that drive them. This approach will be important as studies expand into materials systems with a variety of boundary conditions. It has been reported that the lattice mismatch between the film and substrate leads to a biaxialstrain mechanical boundary condition ${ }^{170-173}$, which can be altered by choosing substrates spanning a wide range of lattice parameters and can reach up to several percent ${ }^{62,174-177}$. Such alterations of mechanical boundary conditions allow the polarization of the thin films to be tuned via a strong polarization-strain coupling $170,173,178,179$ and also result in the formation of ferroelastic-ferroelectric domain patterns to release the biaxial strain ${ }^{180-183}$. Phases that do not exist in bulk can also be stabilized in thin films ${ }^{176,177,184-186}$. For example, when BFO is grown on a highly mismatched (high-strain) substrate, such as LAO, the film can adopt a mix of tetragonal-like and rhombohedral-like phases $^{177}$; however, when grown on a low-strain substrate like TSO, the film adopts only the rhombohedral-like phase ${ }^{187}$. In addition, electrical boundary conditions are critically dependent on the free charge compensation at the interfaces (or surfaces) and can be tailored by choosing substrates or epitaxial buffer layers with different conductivities ${ }^{188,189}$. Additional restrictions on the boundary condition can be made by changing the substrates' vicinity or atomic termination ${ }^{190,191}$. Based on these studies, it is clear that the boundary conditions will strongly influence the interfacial properties of ferroelectric thin films and having highresolution characterization methods that can determine the local polarization and electronic properties at atomically sharp interfaces, such as STEM imaging, EELS, 4D-STEM, and SPM, will play an important role in fully characterizing these films.

\section{Summary}

In order to keep up with the exponentially growing need for computing efficiency, nontraditional materials such as twodimensional materials and beyond-CMOS logic structures relying on multiple order parameters such as charge, spin, and lattice, have become plausible ways to significantly improve the energy efficiency and speed of integrated circuits. Computing with spintronics and multiferroics has the advantage of collective switching, storing thresholding behavior, and non-volatility ${ }^{192,193}$. We have reviewed some of the most recent developments in polarization-controlled interface properties, showing that polarization can be used to control these order parameters. In particular, polarization-induced ferromagnetism enriches the magnetoelectric coupling family. The development of electronics technology requires smaller size and lower power consumption, continuing to spur the research boom in ultrathin or 2D ferroelectrics, which have promising characteristics, such as improved carrier transport and reduced dynamic energy $96,99,194,195$. For the same reason, enhancing the polarization of ultrathin films has become very important. In general, strain engineering and defect engineering can be used to optimize the polarization properties ${ }^{64,66,196-198}$. The introduction of freestanding films also opens the door for many avenues for polarization enhancement to be explored as the thin film can be transferred to any substrate ${ }^{77}$. This work on ferroelectric films and their characterization significantly expands the design space and promotes the exploration of diverse degrees of freedom, revealing more cross-cutting possibilities.

We reviewed the emergent phenomena at two kinds of interfaces (heterointerface and homo-interface) controlled by ferroelectric polarization. As Hebert Kroemer has said that "the interface is the device ${ }^{\prime 199}$, and these interfaces provide vast and unforeseen opportunities to create devices with interesting properties. In addition, with the development of state-of-the-art TEM techniques, the gap between experimental observation and theoretical modeling has never become so seamless. 
The combination of the two techniques has become a routine in designing and evaluating nanodevices by correlating their structures with physical properties. We hope that this review can provide the readers with a different perspective towards the understanding of how polarization is involved in some of the emergent interfacial phenomena.

Received: 11 March 2021; Accepted: 5 July 2021; Published online: 16 August 2021

\section{REFERENCES}

1. Ramesh, R. Thin Film Ferroelectric Materials and Devices (Springer Press, New York, 2013).

2. Spaldin, N. A., Cheong, S.-W. \& Ramesh, R. Multiferroics: past, present, and future. Phys. Today 63, 38-43 (2010).

3. Lee, J. H. et al. A strong ferroelectric ferromagnet created by means of spin-lattice coupling. Nature 466, 954-958 (2010).

4. Bristowe, N., Varignon, J., Fontaine, D., Bousquet, E. \& Ghosez, P. Ferromagnetism induced by entangled charge and orbital orderings in ferroelectric titanate perovskites. Nat. Commun. 6, 1-6 (2015).

5. Damjanovic, D. Ferroelectric, dielectric and piezoelectric properties of ferroelectric thin films and ceramics. Rep. Prog. Phys. 61, 1267-1324 (1998)

6. Choi, T., Lee, S., Choi, Y. J., Kiryukhin, V. \& Cheong, S.-W. Switchable ferroelectric diode and photovoltaic effect in $\mathrm{BiFeO}_{3}$. Science 324, 63-66 (2009).

7. Gruverman, A. \& Kholkin, A. Nanoscale ferroelectrics: processing, characterization and future trends. Rep. Prog. Phys. 69, 2443-2474 (2005).

8. Scott, J. F. Ferroelectric Memories (Springer Press, Berlin Heidelberg, 2000).

9. Catalan, G. \& Scott, J. F. Physics and applications of bismuth ferrite. Adv. Mater 21, 2463-2485 (2009).

10. Lancaster, M., Powell, J. \& Porch, A. Thin-film ferroelectric microwave devices Supercond. Sci. Technol. 11, 1323-1334 (1998).

11. Dawber, M., Rabe, K. \& Scott, J. Physics of thin-film ferroelectric oxides. Rev. Mod. Phys. 77, 1083-1130 (2005).

12. Bert, J. A. et al. Direct imaging of the coexistence of ferromagnetism and superconductivity at the $\mathrm{LaAlO}_{3} / \mathrm{SrTiO}_{3}$ interface. Nat. Phys. 7, 767-771 (2011).

13. Reyren, N. et al. Superconducting interfaces between insulating oxides. Science 317, 1196-1199 (2007).

14. Gruverman, A. et al. Tunneling electroresistance effect in ferroelectric tunnel junctions at the nanoscale. Nano Lett. 9, 3539-3543 (2009).

15. Wen, Z., Li, C., Wu, D., Li, A. \& Ming, N. Ferroelectric-field-effect-enhanced electroresistance in metal/ferroelectric/semiconductor tunnel junctions. Nat. Mater. 12, 617-621 (2013)

16. Chi, X. et al. Unusual hole and electron midgap states and orbital reconstructions induced huge ferroelectric tunneling electroresistance in $\mathrm{BaTiO}_{3} / \mathrm{SrTiO}_{3}$. Nano Lett. 20, 1101-1109 (2020).

17. Ohtomo, A. \& Hwang, H. A high-mobility electron gas at the $\mathrm{LaAlO}_{3} / \mathrm{SrTiO}_{3}$ heterointerface. Nature 427, 423-426 (2004).

18. Tsymbal, E. Y., Dagotto, E. R., Eom, C.-B. \& Ramesh, R. Multifunctional Oxide Heterostructures (Oxford Univ. Press, Oxford, 2012).

19. Crawford, J. \& English, F. Ceramic ferroelectric field effect studies. IEEE Trans. Electron Devices 16, 525-532 (1969).

20. Pantel, D., Goetze, S., Hesse, D. \& Alexe, M. Reversible electrical switching of spin polarization in multiferroic tunnel junctions. Nat. Mater. 11, 289-293 (2012).

21. Song, H.-D. et al. Electrical control of magnetic proximity effect in a graphene/ multiferroic heterostructure. Appl. Phys. Lett. 113, 183101 (2018)

22. Ahn, C. et al. Ferroelectric field effect in ultrathin $\mathrm{SrRuO}_{3}$ films. Appl. Phys. Lett 70, 206-208 (1997).

23. Takahashi, K. et al. Local switching of two-dimensional superconductivity using the ferroelectric field effect. Nature 441, 195-198 (2006).

24. Kim, Y.-M. et al. Direct observation of ferroelectric field effect and vacancycontrolled screening at the $\mathrm{BiFeO}_{3} / \mathrm{La}_{x} \mathrm{Sr}_{1-\mathrm{x}} \mathrm{MnO}_{3}$ interface. Nat. Mater. 13, 1019-1025 (2014).

25. Brinkman, A. et al. Magnetic effects at the interface between non-magnetic oxides. Nat. Mater. 6, 493-496 (2007)

26. Salluzzo, M. et al. Origin of interface magnetism in $\mathrm{BiMnO}_{3} / \mathrm{SrTiO}_{3}$ and $\mathrm{LaAlO}_{3} /$ $\mathrm{SrTiO}_{3}$ heterostructures. Phys. Rev. Lett. 111, 087204 (2013).

27. Gregg, J. M. \& Kumar, A. Trawling for complements. Nature 510, 481-482 (2014).

28. Seidel, J. et al. Domain wall conductivity in La-doped $\mathrm{BiFeO}_{3}$. Phys. Rev. Lett. 105 197603 (2010).

29. Rojac, T. et al. Domain-wall conduction in ferroelectric $\mathrm{BiFeO}_{3}$ controlled by accumulation of charged defects. Nat. Mater. 16, 322-327 (2017).
30. Vasudevan, R. K. et al. Domain wall conduction and polarization-mediated transport in ferroelectrics. Adv. Funct. Mater. 23, 2592-2616 (2013).

31. Sluka, T., Tagantsev, A. K., Bednyakov, P. \& Setter, N. Free-electron gas at charged domain walls in insulating $\mathrm{BaTiO}_{3}$. Nat. Commun. 4, 1-6 (2013).

32. Seidel, J. et al. Domain wall functionality in $\mathrm{BiFeO}_{3}$. Phase Transit. 86, 53-66 (2013).

33. Campbell, M. et al. Hall effect in charged conducting ferroelectric domain walls. Nat. Commun. 7, 1-6 (2016).

34. Gareeva, Z., Diéguez, O., Iniguez, J. \& Zvezdin, A. K. Complex domain walls in $\mathrm{BiFeO}_{3}$. Phys. Rev. B 91, 060404 (2015).

35. Yang, Z., Lange, M., Volodin, A., Szymczak, R. \& Moshchalkov, V. V. Domain-wall superconductivity in superconductor-ferromagnet hybrids. Nat. Mater. 3, 793-798 (2004).

36. Buzdin, A. I. \& Mel'nikov, A. S. Domain wall superconductivity in ferromagnetic superconductors. Phys. Rev. B 67, 020503 (2003).

37. Kim, S. I. et al. Non-volatile control of $2 D E G$ conductivity at oxide interfaces. $A d v$. Mater. 25, 4612-4617 (2013).

38. Cantoni, $C$. et al. Electron transfer and ionic displacements at the origin of the 2D electron gas at the LAO/STO interface: direct measurements with atomiccolumn spatial resolution. Adv. Mater. 24, 3952-3957 (2012).

39. Bark, C. et al. Tailoring a two-dimensional electron gas at the $\mathrm{LaAlO}_{3} / \mathrm{SrTiO}_{3}$ (001) interface by epitaxial strain. PNAS 108, 4720-4724 (2011).

40. Mix, C., Finizio, S., Kläui, M. \& Jakob, G. Conductance control at the $\mathrm{LaAlO}_{3} /$ $\mathrm{SrTiO}_{3}$-interface by a multiferroic $\mathrm{BiFeO}_{3}$ ad-layer. Appl. Phys. Lett. 104, 262903 (2014).

41. Pallecchi, I. et al. Seebeck effect in the conducting $\mathrm{LaAlO}_{3} / \mathrm{SrTiO}_{3}$ interface. Phys. Rev. B 81, 085414 (2010).

42. Li, L., Richter, C., Mannhart, J. \& Ashoori, R. Coexistence of magnetic order and two-dimensional superconductivity at $\mathrm{LaAlO}_{3} / \mathrm{SrTiO}_{3}$ interfaces. Nat. Phys. 7, 762-766 (2011).

43. Nakagawa, N., Hwang, H. Y. \& Muller, D. A. Why some interfaces cannot be sharp Nat. Mater. 5, 204-209 (2006).

44. Herranz, G. et al. High mobility in $\mathrm{LaAlO}_{3} / \mathrm{SrTiO}_{3}$ heterostructures: Origin, dimensionality, and perspectives. Phys. Rev. Lett. 98, 216803 (2007).

45. Siemons, W. et al. Origin of charge density at $\mathrm{LaAlO}_{3}$ on $\mathrm{SrTiO}_{3}$ heterointerfaces: Possibility of intrinsic doping. Phys. Rev. Lett. 98, 196802 (2007).

46. Zhang, F. et al. Dynamic modulation of the transport properties of the $\mathrm{LaAlO}_{3} /$ $\mathrm{SrTiO}_{3}$ interface using uniaxial strain. Phys. Rev. B 93, 214427 (2016).

47. Jia, C.-L. et al. Unit-cell scale mapping of ferroelectricity and tetragonality in epitaxial ultrathin ferroelectric films. Nat. Mater. 6, 64-69 (2007).

48. Zhang, Y. et al. Anisotropic polarization-induced conductance at a ferroelectric-insulator interface. Nat. Nanotechnol. 13, 1132-1136 (2018).

49. Hausmann, S. et al. Atomic-scale engineering of ferroelectric-ferromagnetic interfaces of epitaxial perovskite films for functional properties. Sci. Rep. 7, 1-16 (2017).

50. Bocher, L. et al. Atomic and electronic structure of the $\mathrm{BaTiO}_{3} / \mathrm{Fe}$ interface in multiferroic tunnel junctions. Nano Lett. 12, 376-382 (2012).

51. Addiego, C., Gao, W. \& Pan, X. Thickness and defocus dependence of interatomic electric fields measured by scanning diffraction. Ultramicroscopy $\mathbf{2 0 8}$, 112850 (2020).

52. Okunishi, E. et al. Visualization of light elements at ultrahigh resolution by STEM annular bright field microscopy. Microsc. Microanal. 15, 164-165 (2009).

53. Nelson, C. T. et al. Domain dynamics during ferroelectric switching. Science 334 968-971 (2011).

54. Gao, P. et al. Revealing the role of defects in ferroelectric switching with atomic resolution. Nat. Commun. 2, 1-6 (2011).

55. Gao, P. et al. Direct observations of retention failure in ferroelectric memories. Adv. Mater. 24, 1106-1110 (2012).

56. Gao, P. et al. Atomic-scale mechanisms of ferroelastic domain-wall-mediated ferroelectric switching. Nat. Commun. 4, 1-9 (2013).

57. Gao, P. et al. Ferroelastic domain switching dynamics under electrical and mechanical excitations. Nat. Commun. 5, 1-8 (2014).

58. Smith, D. J. et al. Recent studies of oxide-semiconductor heterostructures using aberration-corrected scanning transmission electron microscopy. J. Mater. Res. 32, 912-920 (2017).

59. Li, L. et al. Defect-induced hedgehog polarization states in multiferroics. Phys. Rev. Lett. 120, 137602 (2018).

60. Li, L. et al. Atomic-scale mechanisms of defect-induced retention failure in ferroelectrics. Nano Lett. 17, 3556-3562 (2017).

61. Li, L. et al. Giant resistive switching via control of ferroelectric charged domain walls. Adv. Mater. 28, 6574-6580 (2016).

62. Nelson, C. T. et al. Spontaneous vortex nanodomain arrays at ferroelectric heterointerfaces. Nano Lett. 11, 828-834 (2011).

63. Yadav, A. et al. Observation of polar vortices in oxide superlattices. Nature $\mathbf{5 3 0}$ 198-201 (2016) 
64. Li, L. et al. Observation of strong polarization enhancement in ferroelectric tunnel junctions. Nano Lett. 19, 6812-6818 (2019).

65. Li, L. et al. Control of domain structures in multiferroic thin films through defect engineering. Adv. Mater. 30, 1802737 (2018).

66. Xie, L. et al. Giant ferroelectric polarization in ultrathin ferroelectrics via boundary-condition engineering. Adv. Mater. 29, 1701475 (2017).

67. MacLaren, I. \& Ramasse, Q. M. Aberration-corrected scanning transmission electron microscopy for atomic-resolution studies of functional oxides. Int. Mater. Rev. 59, 115-131 (2014).

68. Zhang, Y., Sun, J., Perdew, J. P. \& Wu, X. Comparative first-principles studies of prototypical ferroelectric materials by LDA, GGA, and SCAN meta-GGA. Phys. Rev. B 96, 035143 (2017).

69. Wang, J.-J., Wang, B. \& Chen, L.-Q. Understanding, predicting, and designing ferroelectric domain structures and switching guided by the phase-field method. Annu. Rev. Mater. Res. 49, 127-152 (2019).

70. Liu, S., Grinberg, I. \& Rappe, A. M. Intrinsic ferroelectric switching from first principles. Nature 534, 360-363 (2016).

71. Rabe, K. M. \& Ghosez, P. First-principles studies of ferroelectric oxides. Phys. Ferroelectr. 105, 117-174 (2007).

72. Andreoni, W. \& Yip, S. Handbook of Materials Modeling: Applications: Current and Emerging Materials (Springer Press, 2020).

73. Gao, W. et al. Real-space charge-density imaging with sub-ångström resolution by four-dimensional electron microscopy. Nature 575, 480-484 (2019).

74. Shibata, N. et al. Differential phase-contrast microscopy at atomic resolution. Nat. Phys. 8, 611-615 (2012).

75. Müller-Caspary, K. et al. Electrical polarization in AIN/GaN nanodisks measured by momentum-resolved 4D scanning transmission electron microscopy. Phys. Rev. Lett. 122, 106102 (2019).

76. Yadav, A. K. et al. Spatially resolved steady-state negative capacitance. Nature 565, 468-471 (2019).

77. Ji, D. et al. Freestanding crystalline oxide perovskites down to the monolayer limit. Nature 570, 87-90 (2019).

78. Wu, S.-Y. A new ferroelectric memory device, metal-ferroelectric-semiconductor transistor. IEEE Trans. Electron Devices 21, 499-504 (1974).

79. Marshall, M. S. et al. Conduction at a ferroelectric interface. Phys. Rev. Appl. 2, 051001 (2014).

80. Zhang, Y. et al. Discovery of a magnetic conductive interface in $\mathrm{PbZr}_{0.2} \mathrm{Ti}_{0.8} \mathrm{O}_{3} /$ $\mathrm{SrTiO}_{3}$ heterostructures. Nat. Commun. 9, 1-9 (2018).

81. Hwang, H. Y. et al. Emergent phenomena at oxide interfaces. Nat. Mater. 11, 103-113 (2012).

82. Sulpizio, J. A., Ilani, S., Irvin, P. \& Levy, J. Nanoscale phenomena in oxide heterostructures. Annu. Rev. Mater. Res. 44, 117-149 (2014).

83. Chen, $\mathrm{C}$. et al. Two-dimensional electron gas at the Ti-diffused $\mathrm{BiFeO}_{3} / \mathrm{SrTiO}_{3}$ interface. Appl. Phys. Lett. 107, 031601 (2015).

84. Fredrickson, K. D. \& Demkov, A. A. Switchable conductivity at the ferroelectric interface: nonpolar oxides. Phys. Rev. B 91, 115126 (2015).

85. Aguado-Puente, P. et al. Model of two-dimensional electron gas formation at ferroelectric interfaces. Phys. Rev. B 92, 035438 (2015).

86. Zhang, Z., Wu, P., Chen, L. \& Wang, J. First-principles prediction of a two dimensional electron gas at the $\mathrm{BiFeO}_{3} / \mathrm{SrTiO}_{3}$ interface. Appl. Phys. Lett. 99, 062902 (2011)

87. Tao, R. et al. Substantially enhanced robustness of quantum Hall effect in graphene on $\mathrm{LaAlO}_{3} / \mathrm{SrTiO}_{3}$ heterostructure. Appl. Phys. Express 13, 035001 (2020).

88. Matsubara, Y. et al. Observation of the quantum Hall effect in $\delta$-doped $\mathrm{SrTiO}_{3}$. Nat. Commun. 7, 1-7 (2016).

89. Behtash, M., Nazir, S., Wang, Y. \& Yang, K. Polarization effects on the interfacial conductivity in $\mathrm{LaAlO}_{3} / \mathrm{SrTiO}_{3}$ heterostructures: a first-principles study. Phys. Chem. Chem. Phys. 18, 6831-6838 (2016).

90. Tra, V. T. et al. Ferroelectric control of the conduction at the $\mathrm{LaAlO}_{3} / \mathrm{SrTiO}_{3}$ heterointerface. Adv. Mater. 25, 3357-3364 (2013).

91. Kim, S.-I. et al. Giant electroresistive ferroelectric diode on 2DEG. Sci. Rep. 5, 1-7 (2015).

92. Li, Y., Phattalung, S. N., Limpijumnong, S., Kim, J. \& Yu, J. Formation of oxygen vacancies and charge carriers induced in the n-type interface of a $\mathrm{LaAlO}_{3}$ overlayer on $\mathrm{SrTiO}_{3}$ (001). Phys. Rev. B 84, 245307 (2011).

93. Kim, T. L. \& Jang, H. W. Tailoring two-dimensional electron gas conductivity at oxide heterointerfaces. Curr. Appl. Phys. 17, 626-639 (2017).

94. Wang, S. et al. Ferroelectric polarization-modulated interfacial fine structures involving two-dimensional electron gases in $\mathrm{Pb}\left(\mathrm{Zr}, \mathrm{Ti}^{\mathrm{O}} \mathrm{O}_{3} / \mathrm{LaAlO}_{3} / \mathrm{SrTiO}_{3}\right.$ heterostructures. ACS Appl. Mater. Inter. 10, 1374-1382 (2018).

95. Niranjan, M. K., Wang, Y., Jaswal, S. S. \& Tsymbal, E. Y. Prediction of a switchable two-dimensional electron gas at ferroelectric oxide interfaces. Phys. Rev. Lett. 103, 016804 (2009).

96. Mannhart, J., Blank, D. H., Hwang, H., Millis, A. \& Triscone, J.-M. Two-dimensional electron gases at oxide interfaces. MRS Bull. 33, 1027-1034 (2008).
97. Hwang, H. Y. Tuning interface states. Science 313, 1895-1896 (2006).

98. Ramesh, R. \& Spaldin, N. A. Multiferroics: progress and prospects in thin films. Nanosci. Nanotechnol. 6, 20-28 (2010).

99. Zubko, P., Gariglio, S., Gabay, M., Ghosez, P. \& Triscone, J.-M. Interface physics in complex oxide heterostructures. Annu. Rev. Condens. Matter Phys. 2, 141-165 (2011).

100. Ramirez, A. P. Oxide electronics emerge. Science 315, 1377-1378 (2007).

101. Scott, J. Applications of modern ferroelectrics. Science 315, 954-959 (2007).

102. Tsymbal, E. Y., Gruverman, A., Garcia, V., Bibes, M. \& Barthélémy, A. Ferroelectric and multiferroic tunnel junctions. MRS Bull. 37, 138-143 (2012).

103. Garcia, V. et al. Ferroelectric control of spin polarization. Science 327, 1106-1110 (2010).

104. Chen, J. et al. Reversible magnetism transition at ferroelectric oxide heterointerface. Sci. Bull. 65, 2094-2099 (2020).

105. Spurgeon, S. R. et al. Polarization screening-induced magnetic phase gradients at complex oxide interfaces. Nat. Commun. 6, 1-11 (2015).

106. Kourkoutis, L. F., Song, J., Hwang, H. \& Muller, D. Microscopic origins for stabilizing room-temperature ferromagnetism in ultrathin manganite layers. PNAS 107, 11682-11685 (2010)

107. Li, X., Lindfors-Vrejoiu, I., Ziese, M., Gloter, A. \& Van Aken, P. A. Impact of interfacial coupling of oxygen octahedra on ferromagnetic order in $\mathrm{La}_{0.7} \mathrm{Sr}_{0.3} \mathrm{MnO}_{3} / \mathrm{SrTiO}_{3}$ heterostructures. Sci. Rep. 7, 1-9 (2017).

108. Feng, $\mathrm{Y}$. et al. Insulating phase at low temperature in ultrathin $\mathrm{La}_{0.8} \mathrm{Sr}_{0.2} \mathrm{MnO}_{3}$ films. Sci. Rep. 6, 1-9 (2016).

109. Sharma, P. et al. Nonvolatile ferroelectric domain wall memory. Sci. Adv. 3, e1700512 (2017).

110. Rubio-Marcos, F., Del Campo, A., Marchet, P. \& Fernández, J. F. Ferroelectric domain wall motion induced by polarized light. Nat. Commun. 6, 1-9 (2015).

111. $\mathrm{Ma}, \mathrm{J}$. et al. Controllable conductive readout in self-assembled, topologically confined ferroelectric domain walls. Nat. Nanotechnol. 13, 947-952 (2018).

112. Parkin, S. S., Hayashi, M. \& Thomas, L. Magnetic domain-wall racetrack memory. Science 320, 190-194 (2008).

113. Venkatesan, R. et al (eds.) Proc. 2012 ACM/IEEE International Symposium on Low Power Electronics and Design 185-190 (2012).

114. Catalan, G., Seidel, J., Ramesh, R. \& Scott, J. F. Domain wall nanoelectronics. Rev. Mod. Phys. 84, 119-156 (2012).

115. Körbel, S., Hlinka, J. \& Sanvito, S. Electron trapping by neutral pristine ferroelectric domain walls in $\mathrm{BiFeO}_{3}$. Phys. Rev. B 98, 100104 (2018).

116. Nataf, G. et al. Domain-wall engineering and topological defects in ferroelectric and ferroelastic materials. Nat. Rev. Phys. 2, 634-648 (2020).

117. $\mathrm{Li}, \mathrm{L}$. et al. Atomic scale structure changes induced by charged domain walls in ferroelectric materials. Nano Lett. 13, 5218-5223 (2013).

118. Maksymovych, P. et al. Tunable metallic conductance in ferroelectric nanodomains. Nano Lett. 12, 209-213 (2011).

119. Crassous, A., Sluka, T., Tagantsev, A. K. \& Setter, N. Polarization charge as a reconfigurable quasi-dopant in ferroelectric thin films. Nat. Nanotechnol. 10, 614-618 (2015).

120. Wu, W., Horibe, Y., Lee, N., Cheong, S.-W. \& Guest, J. Conduction of topologically protected charged ferroelectric domain walls. Phys. Rev. Lett. 108, 077203 (2012).

121. Tian, G. et al. Manipulation of conductive domain walls in confined ferroelectric nanoislands. Adv. Funct. Mater. 29, 1807276 (2019).

122. Liu, L. et al. Giant domain wall conductivity in self-assembled $\mathrm{BiFeO}_{3}$ nanocrystals. Adv. Funct. Mater. 31, 2005876 (2021).

123. Seidel, J. et al. Conduction at domain walls in oxide multiferroics. Nat. Mater. 8 , 229-234 (2009).

124. Vasudevan, R. et al. Domain wall geometry controls conduction in ferroelectrics. Nano Lett. 12, 5524-5531 (2012).

125. Kim, D. J., Connell, J., Seo, S. \& Gruverman, A. Domain wall conductivity in semiconducting hexagonal ferroelectric $\mathrm{TbMnO}_{3}$ thin films. Nanotechnology 27, 155705 (2016).

126. Stolichnov, I. et al. Bent ferroelectric domain walls as reconfigurable metallic-like channels. Nano Lett. 15, 8049-8055 (2015).

127. Liu, S. et al. Ferroelectric domain wall induced band gap reduction and charge separation in organometal halide perovskites. J. Phys. Chem. Lett. 6, 693-699 (2015).

128. Qian, C.-X. et al. Domain wall conduction in calcium-modified lead titanate for polarization tunable photovoltaic devices. Cell Rep. Phys. Sci. 1, 100043 (2020).

129. Chiu, Y. P. et al. Atomic-scale evolution of local electronic structure across multiferroic domain walls. Adv. Mater. 23, 1530-1534 (2011).

130. Evans, D. M., Garcia, V., Meier, D. \& Bibes, M. Domains and domain walls in multiferroics. Phys. Sci. Rev. 5, 67 (2020).

131. Eliseev, E. A. et al. Conductivity of twin-domain-wall/surface junctions in ferroelastics: Interplay of deformation potential, octahedral rotations, improper ferroelectricity, and flexoelectric coupling. Phys. Rev. B 86, 085416 (2012). 
132. Farokhipoor, S. \& Noheda, B. Conduction through 71 domain walls in $\mathrm{BiFeO}_{3}$ thin films. Phys. Rev. Lett. 107, 127601 (2011).

133. Lubk, A., Gemming, S. \& Spaldin, N. First-principles study of ferroelectric domain walls in multiferroic bismuth ferrite. Phys. Rev. B 80, 104110 (2009).

134. Zhang, Y. et al. Intrinsic conductance of domain walls in $\mathrm{BiFeO}_{3}$. Adv. Mater. 31 , 1902099 (2019).

135. Bednyakov, P. S., Sturman, B. I., Sluka, T., Tagantsev, A. K. \& Yudin, P. V. Physics and applications of charged domain walls. Npj Comput. Mater. 4, 1-11 (2018).

136. Sifuna, J., García-Fernández, P., Manyali, G. S., Amolo, G. \& Junquera, J. Firstprinciples study of two-dimensional electron and hole gases at the head-tohead and tail-to-tail 180 domain walls in $\mathrm{PbTiO}_{3}$ ferroelectric thin films. Phys. Rev. B 101, 174114 (2020).

137. Gong, J. et al. Interactions of charged domain walls and oxygen vacancies in $\mathrm{BaTiO}_{3}$ : a first-principles study. Mater. Today Phys. 6, 9-21 (2018).

138. Morozovska, A. N., Vasudevan, R. K., Maksymovych, P., Kalinin, S. V. \& Eliseev, E. A. Anisotropic conductivity of uncharged domain walls in $\mathrm{BiFeO}_{3}$. Phys. Rev. $B$ 86, 085315 (2012).

139. Maksymovych, P. et al. Dynamic conductivity of ferroelectric domain walls in $\mathrm{BiFeO}_{3}$. Nano Lett. 11, 1906-1912 (2011).

140. Catalan, G. On the link beween octahedral rotations and conductivity in the domain walls of $\mathrm{BiFeO}_{3}$. Ferroelectrics 433, 65-73 (2012).

141. Diéguez, O., Aguado-Puente, P., Junquera, J. \& Íniguez, J. Domain walls in a perovskite oxide with two primary structural order parameters: first-principles study of $\mathrm{BiFeO}_{3}$. Phys. Rev. B 87, 024102 (2013).

142. Wang, Y. et al. BiFeO 3 domain wall energies and structures: a combined experimental and density functional theory $+U$ study. Phys. Rev. Lett. 110, 267601 (2013)

143. $\mathrm{Lu}, \mathrm{H}$. et al. Electrical tunability of domain wall conductivity in $\mathrm{LiNbO}_{3}$ thin films. Adv. Mater. 31, 1902890 (2019).

144. Kalinin, S. V., Rar, A. \& Jesse, S. A decade of piezoresponse force microscopy: progress, challenges, and opportunities. IEEE Trans. Ultrason Ferroelectr. Freq. Control 53, 2226-2252 (2006).

145. Ponath, $\mathrm{P}$. et al. Carrier density modulation in a germanium heterostructure by ferroelectric switching. Nat. Commun. 6, 1-7 (2015).

146. Li, L., Xie, L. \& Pan, X. Real-time studies of ferroelectric domain switching: a review. Rep. Prog. Phys. 82, 126502 (2019).

147. Lazić, I., Bosch, E. G. \& Lazar, S. Phase contrast STEM for thin samples: Integrated differential phase contrast. Ultramicroscopy 160, 265-280 (2016).

148. Findlay, S. et al. Imaging low Z materials in crystalline environments via scanning transmission electron microscopy. Microsc. Microanal. 25, 1732-1733 (2019).

149. Yücelen, E., Lazić, I. \& Bosch, E. G. Phase contrast scanning transmission electron microscopy imaging of light and heavy atoms at the limit of contrast and resolution. Sci. Rep. 8, 1-10 (2018).

150. Carlsson, A., Alexandrou, I., Yücelen, E., Bosch, E. G. \& Lazić, I. Low dose imaging using simultaneous iDPC-and ADF-STEM for beam sensitive crystalline structures. Microsc. Microanal. 24, 122-123 (2018).

151. de Graaf, S., Momand, J., Mitterbauer, C., Lazar, S. \& Kooi, B. J. Resolving hydrogen atoms at metal-metal hydride interfaces. Sci. Adv. 6, 1-8 (2020).

152. Campanini, M. et al. Imaging and quantification of charged domain walls in $\mathrm{BiFeO}_{3}$. Nanoscale 12, 9186-9193 (2020).

153. Shibata, N. et al. Direct visualization of local electromagnetic field structures by scanning transmission electron microscopy. Acc. Chem. Res. 50, 1502-1512 (2017).

154. Haider, M., Epstein, A., Jarron, P. \& Boulin, C. A versatile, software configurable multichannel STEM detector for angle-resolved imaging. Ultramicroscopy 54, 41-59 (1994).

155. Tate, M. W. et al. High dynamic range pixel array detector for scanning transmission electron microscopy. Microsc. Microanal. 22, 237-249 (2016).

156. Ophus, C. Four-dimensional scanning transmission electron microscopy (4DSTEM): from scanning nanodiffraction to ptychography and beyond. Microsc. Microanal. 25, 563-582 (2019).

157. Müller, K. et al. Atomic electric fields revealed by a quantum mechanical approach to electron picodiffraction. Nat. Commun. 5, 1-8 (2014).

158. Li, X. et al. Manifold learning of four-dimensional scanning transmission electron microscopy. Npj Comput. Mater. 5, 1-8 (2019).

159. Gammer, C., Ozdol, V. B., Liebscher, C. H. \& Minor, A. M. Diffraction contrast imaging using virtual apertures. Ultramicroscopy 155, 1-10 (2015).

160. Wang, Y. et al. Correcting the linear and nonlinear distortions for atomically resolved STEM spectrum and diffraction imaging. Microscopy 67, i114-i122 (2018).

161. Watanabe, M. \& Williams, D. Development of diffraction imaging for orientation analysis of grains in scanning transmission electron microscopy. Microsc. Microanal. 13, 962-963 (2007).
162. Kobler, A., Kashiwar, A., Hahn, H. \& Kübel, C. Combination of in situ straining and ACOM TEM: a novel method for analysis of plastic deformation of nanocrystalline metals. Ultramicroscopy 128, 68-81 (2013).

163. Clément, L., Pantel, R., Kwakman, L. T. \& Rouvière, J. Strain measurements by convergent-beam electron diffraction: the importance of stress relaxation in lamella preparations. Appl. Phys. Lett. 85, 651-653 (2004).

164. Zhang, P. et al. Direct strain measurement in a $65 \mathrm{~nm}$ node strained silicon transistor by convergent-beam electron diffraction. Appl. Phys. Lett. 89, 161907 (2006).

165. Midgley, P. A. Electronic bonding revealed by electron diffraction. Science 331, 1528-1529 (2011)

166. Zuo, J.-M., Kim, M., O'keeffe, M. \& Spence, J. Direct observation of d-orbital holes and $\mathrm{Cu}-\mathrm{Cu}$ bonding in $\mathrm{Cu}_{2} \mathrm{O}$. Nature 401, 49-52 (1999).

167. Nakashima, P. N., Smith, A. E., Etheridge, J., \& Muddle, B. C. The bonding electron density in aluminum. Science 331, 1583-1586 (2011).

168. Müller-Caspary, K. et al. Measurement of atomic electric fields and charge densities from average momentum transfers using scanning transmission electron microscopy. Ultramicroscopy 178, 62-80 (2017).

169. Beyer, A. et al. Quantitative characterization of nanometer-scale electric fields via momentum-resolved STEM. Nano Lett. 21, 2018-2025 (2021).

170. Schlom, D. G. et al. Strain tuning of ferroelectric thin films. Annu. Rev. Mater. Res. 37, 589-626 (2007).

171. Choi, K. J. et al. Enhancement of ferroelectricity in strained $\mathrm{BaTiO}_{3}$ thin films. Science 306, 1005-1009 (2004).

172. Ederer, C. \& Spaldin, N. A. Effect of epitaxial strain on the spontaneous polarization of thin film ferroelectrics. Phys. Rev. Lett. 95, 257601 (2005).

173. Schlom, D. G. et al. Elastic strain engineering of ferroic oxides. MRS Bull. 39, 118-130 (2014).

174. Adamo, C. et al. Effect of biaxial strain on the electrical and magnetic properties of (001) $\mathrm{La}_{0.7} \mathrm{Sr}_{0.3} \mathrm{MnO}_{3}$ thin films. Appl. Phys. Lett. 95, 112504 (2009).

175. Infante, I. C. et al. Bridging multiferroic phase transitions by epitaxial strain in $\mathrm{BiFeO}_{3}$. Phys. Rev. Lett. 105, 057601 (2010)

176. Béa, $\mathrm{H}$. et al. Evidence for room-temperature multiferroicity in a compound with a giant axial ratio. Phys. Rev. Lett. 102, 217603 (2009).

177. Zeches, R. et al. A strain-driven morphotropic phase boundary in $\mathrm{BiFeO}_{3}$. Science 326, 977-980 (2009).

178. Haeni, J. et al. Room-temperature ferroelectricity in strained $\mathrm{SrTiO}_{3}$. Nature 430 758-761 (2004).

179. Cao, J. \& Wu, J. Strain effects in low-dimensional transition metal oxides. Mater. Sci. Eng. R. Rep. 71, 35-52 (2011).

180. Lee, K. \& Baik, S. Ferroelastic domain structure and switching in epitaxial ferroelectric thin films. Annu. Rev. Mater. Res. 36, 81-116 (2006).

181. Zavaliche, F. et al. Ferroelectric domain structure in epitaxial $\mathrm{BiFeO}_{3}$ films. Appl. Phys. Lett. 87, 182912 (2005).

182. Chen, Y. et al. Ferroelectric domain structures of epitaxial (001) $\mathrm{BiFeO}_{3}$ thin films. Appl. Phys. Lett. 90, 072907 (2007)

183. Streiffer, S. et al. Domain patterns in epitaxial rhombohedral ferroelectric films. I. Geometry and experiments. J. Appl. Phys. 83, 2742-2753 (1998).

184. Mazumdar, D. et al. Nanoscale switching characteristics of nearly tetragonal $\mathrm{BiFeO}_{3}$ thin films. Nano Lett. 10, 2555-2561 (2010).

185. Damodaran, A. R. et al. Nanoscale structure and mechanism for enhanced electromechanical response of highly strained $\mathrm{BiFeO} 3$ thin films. Adv. Mater. 23, 3170-3175 (2011).

186. Zhang, J. et al. Microscopic origin of the giant ferroelectric polarization in tetragonal-like $\mathrm{BiFeO}_{3}$. Phys. Rev. Lett. 107, 147602 (2011).

187. Folkman, C. et al. Stripe domain structure in epitaxial (001) $\mathrm{BiFeO}_{3}$ thin films on orthorhombic $\mathrm{TbSCO}_{3}$ substrate. Appl. Phys. Lett. 94, 251911 (2009).

188. Chisholm, M. F., Luo, W., Oxley, M. P., Pantelides, S. T. \& Lee, H. N. Atomic-scale compensation phenomena at polar interfaces. Phys. Rev. Lett. 105, 197602 (2010).

189. Lichtensteiger, C., Fernandez-Pena, S., Weymann, C., Zubko, P. \& Triscone, J.-M. Tuning of the depolarization field and nanodomain structure in ferroelectric thin films. Nano Lett. 14, 4205-4211 (2014).

190. Chu, Y. H. et al. Domain control in multiferroic $\mathrm{BiFeO}_{3}$ through substrate vicinality. Adv. Mater. 19, 2662-2666 (2007)

191. Jang, H. W. et al. Domain engineering for enhanced ferroelectric properties of epitaxial (001) BiFeO thin films. Adv. Mater. 21, 817-823 (2009).

192. Spaldin, N. A. Multiferroics: past, present, and future. MRS Bull. 42, 385-390 (2017).

193. Manipatruni, S., Nikonov, D. E. \& Young, I. A. Beyond CMOS computing with spin and polarization. Nat. Phys. 14, 338-343 (2018)

194. Mannhart, J. \& Schlom, D. Oxide interfaces-an opportunity for electronics. Science 327, 1607-1611 (2010).

195. Huijben, M. et al. Structure-property relation of $\mathrm{SrTiO}_{3} / \mathrm{LaAlO}_{3}$ interfaces. Adv. Mater. 21, 1665-1677 (2009). 
196. Wang, J. et al. Epitaxial $\mathrm{BiFeO}_{3}$ multiferroic thin film heterostructures. science 299, 1719-1722 (2003)

197. Lu, H. et al. Enhancement of ferroelectric polarization stability by interface engineering. Adv. Mater. 24, 1209-1216 (2012).

198. Chen, C. et al. Controllable defect driven symmetry change and domain structure evolution in $\mathrm{BiFeO}_{3}$ with enhanced tetragonality. Nanoscale 11, 8110-8118 (2019).

199. Kroemer, H. Nobel Lecture: Quasielectric fields and band offsets: teaching electrons new tricks. RMP 73, 783 (2001).

200. Song, B. et al. Hollow electron ptychographic diffractive imaging. Phys. Rev. Lett. 121, 146101 (2018).

201. You, L. et al. Enhancing ferroelectric photovoltaic effect by polar order engineering. Sci. Adv. 4, eaat3438 (2018).

\section{ACKNOWLEDGEMENTS}

This work was supported by the Department of Energy, Office of Basic Energy Sciences, Division of Materials Sciences and Engineering under Grant DE-SC0014430, and partially by the National Science Foundation under grant numbers DMR2034738. F.Y. acknowledges the fellowship support from the China Scholarship Council (No. 201806280368) for studies at the University of California, Irvine. The authors acknowledge the use of facilities and instrumentation at the Irvine Materials Research Institute supported in part by the National Science Foundation through the Materials Research Science and Engineering Center program (DMR-2011967).

\section{AUTHOR CONTRIBUTIONS}

X.P. conserved the ideas and directed this project. F.Y., Y.Z., and C.A. prepared the manuscript with contributions from all authors.

\section{COMPETING INTERESTS}

The authors declare no competing interests.

\section{ADDITIONAL INFORMATION}

Correspondence and requests for materials should be addressed to X.P.

Reprints and permission information is available at http://www.nature.com/ reprints

Publisher's note Springer Nature remains neutral with regard to jurisdictional claims in published maps and institutional affiliations.

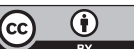

Open Access This article is licensed under a Creative Commons Attribution 4.0 International License, which permits use, sharing, adaptation, distribution and reproduction in any medium or format, as long as you give appropriate credit to the original author(s) and the source, provide a link to the Creative Commons license, and indicate if changes were made. The images or other third party material in this article are included in the article's Creative Commons license, unless indicated otherwise in a credit line to the material. If material is not included in the article's Creative Commons license and your intended use is not permitted by statutory regulation or exceeds the permitted use, you will need to obtain permission directly from the copyright holder. To view a copy of this license, visit http://creativecommons. org/licenses/by/4.0/.

(c) The Author(s) 2021 